\title{
Dense core formation by fragmentation of velocity-coherent filaments in L1517 ${ }^{\star}$
}

\begin{abstract}
A. Hacar and M. Tafalla
Observatorio Astronómico Nacional (IGN), Alfonso XII 3, 28014 Madrid, Spain

e-mail: a.hacar@oan.es

Received 6 April 2011 / Accepted 22 June 2011

\section{ABSTRACT}

Context. Low-mass star-forming cores differ from their surrounding molecular cloud in turbulence, shape, and density structure. Aims. We aim to understand how dense cores form out of the less dense cloud material by studying the connection between these two regimes.

Methods. We observed the $\mathrm{L} 1517$ dark cloud in $\mathrm{C}^{18} \mathrm{O}(1-0), \mathrm{N}_{2} \mathrm{H}^{+}(J=1-0)$, and $\mathrm{SO}\left(J_{\mathrm{N}}=3_{2}-2_{1}\right)$ with the FCRAO $14 \mathrm{~m}$ telescope, and in the $1.2 \mathrm{~mm}$ dust continuum with the IRAM $30 \mathrm{~m}$ telescope.

Results. Most of the gas in the cloud lies in four filaments that have typical lengths of $0.5 \mathrm{pc}$. Five starless cores are embedded in these filaments and have chemical compositions indicative of different evolutionary stages. The filaments have radial profiles of $\mathrm{C}^{18} \mathrm{O}(1-0)$ emission with a central flattened region and a power-law tail, and can be fitted approximately as isothermal, pressure-supported cylinders. The filaments, in addition, are extremely quiescent. They have subsonic internal motions and are coherent in velocity over their whole length. The large-scale motions in the filaments can be used to predict the velocity inside the cores, indicating that core formation has not decoupled the dense gas kinematically from its parental material. In two filaments, these large-scale motions consist of oscillations in the velocity centroid, and a simple kinematic model suggests that they may be related to core-forming flows. Conclusions. Core formation in L1517 seems to have occurred in two steps. First, the subsonic, velocity-coherent filaments have condensed out of the more turbulent ambient cloud. Then, the cores fragmented quasi-statically and inherited the kinematics of the filaments. Turbulence dissipation has therefore occurred mostly on scales on the order of $0.5 \mathrm{pc}$ or larger, and seems to have played a small role in the formation of the individual cores.
\end{abstract}

Key words. ISM: clouds - ISM: molecules - ISM: kinematics and dynamics - ISM: structure - stars: formation - radio lines: ISM

\section{Introduction}

Dense cores in nearby dark clouds are the birth sites of solarmass stars, and they represent the simplest environments where stars can be formed. The study of the internal structure of these cores offers a unique opportunity to determine the initial conditions of low-mass star formation, and for this reason an intense observational effort is under way to characterize cores in detail (see recent reviews by di Francesco et al. 2007; Ward-Thompson et al. 2007; Bergin \& Tafalla 2007).

Compared to their turbulent parent clouds dense cores are very quiescent. Molecular line observations in tracers like ammonia reveal that the gas in a dense core has subsonic internal motions, indicating that thermal pressure contributes more than turbulence in providing support against gravity (Myers 1983). In addition, the gas motions inside a core are "coherent", in the sense that the observed linewidth does not depend on radius (Goodman et al. 1998), and thus deviates from the linewidth-size relation characteristic of the large-scale gas in a cloud (Larson 1981). Also in contrast to the irregularly shaped cloud gas, cores often display a regular, close-to-spherical geometry (Myers et al. 1991). A number of cores even present centrally concentrated density profiles close to those predicted by hydrostatic equilibrium models (Alves et al. 2001), again suggesting that the core

\footnotetext{
* Based on observations carried out with the FCRAO $14 \mathrm{~m}$ and IRAM $30 \mathrm{~m}$ telescopes. IRAM is supported by INSU/CNRS (France), MPG (Germany) and IGN (Spain).
}

gas conditions differ significantly from those of the cloud gas on larger scales.

How quiescent, centrally-concentrated cores form out of the more turbulent, less-dense cloud material is still a matter of debate. A number of core-formation mechanisms have been proposed over the years, ranging from the quasi-static loss of magnetic support via ambipolar diffusion (Shu et al. 1987; Mouschovias \& Ciolek 1999) to the rapid dissipation of turbulence due to supersonic shocks (Padoan et al. 2001; Klessen et al. 2005; Vázquez-Semadeni et al. 2005). Observationally, it is unclear which of these proposed scenarios can fit the variety of existing constraints. Ambipolar diffusion models, for example, seem to predict stronger magnetic fields than observed (Crutcher et al. 2010), and they usually predict contraction times that significantly exceed those inferred from observed core lifetimes and chemical clocks (Lee et al. 1999; Tafalla et al. 2002). Models of core formation by shocks, on the other hand, predict linewidths and velocity displacements between tracers that are larger than commonly observed in cores (Walsh et al. 2004; Kirk et al. 2007a).

Observational progress in constraining the mechanism of core formation requires probing the connection between the dense cores and the less-dense material that surrounds them. This less-dense material likely represents the gas out of which the cores have condensed, so by comparing its geometry, density, and kinematics with those of the core gas, we could infer the physical changes involved in the formation of a dense 
core. Unfortunately, studying the cloud-to-core transition requires combining observations of tracers sensitive to different density regimes, and until recently, such multi-tracer analysis has been hindered by a number of inconsistencies between the emission from different molecules (Zhou et al. 1989; Lemme et al. 1996).

Work carried out over the past decade has revealed that most inconsistencies between tracers result from chemical changes that occur in the gas at dense-core densities and, in particular, from the selective freeze-out of molecules onto cold dust grains (Kuiper et al. 1996; Caselli et al. 1999; Bergin et al. 2002; Tafalla et al. 2002; Aikawa et al. 2005). As a result of this work, a reasonably consistent picture of the chemical behavior of the different gas tracers has emerged. According to this picture, most carbon-bearing molecules (including CO and CS) disappear from the gas phase at densities close to a few $10^{4} \mathrm{~cm}^{-3}$, while nitrogen-bearing molecules like $\mathrm{NH}_{3}$ and $\mathrm{N}_{2} \mathrm{H}^{+}$survive undepleted up to densities that are at least one order of magnitude higher. In addition, several species like $\mathrm{SO}$ and $\mathrm{C}_{2} \mathrm{~S}$ present significant abundance enhancements at early evolutionary times, but end up freezing out onto the grains at similar densities to the C-bearing species (see Bergin \& Tafalla 2007 for a review of core chemistry).

This new understanding of molecular chemistry at intermediate gas densities now makes it possible to combine observations of different tracers and to finally explore the transition from cloud gas to core material self-consistently. An excellent region for investigating this transition is the L1517 dark cloud in the Taurus-Auriga molecular complex (see Kenyon et al. 2008 for a Taurus overview). L1517 appears in optical images as a region of enhanced obscuration associated with the reflection nebulosity from the young stars AB Aur and SU Aur (Lynds 1962; Strom et al. 1976; Schneider \& Elmegreen 1979). The CO observations of the cloud have shown that the extended gas consists of several filamentary components that extend to the northwest of the nebulosity and occupy a region of about $20^{\prime} \times 10^{\prime}$ coincident with the optical obscuration (Heyer et al. 1987). Embedded in these components lies a collection of several dense cores that are easily distinguished in the optical images thanks to the contrast provided by the bright nebulosity from AB Aur and SU Aur, which lie at least $0.3 \mathrm{pc}$ in projection from the cores (Schneider $\&$ Elmegreen 1979). Radio observations of these cores reveal the physical properties typical of the Taurus-Auriga core population (Benson \& Myers 1989), and a prominent member of the group, L1517B, has been the subject of detailed study owing to its regular shape and clear pattern of molecular freeze out (Tafalla et al. 2004, 2006).

A notable feature of the L1517 cores is that they all appear to be starless (Strom et al. 1976; Beichman et al. 1986; Kirk et al. 2007b). This lack of embedded protostars is probably responsible for the $\mathrm{L} 1517$ cloud presenting some of the narrowest line profiles in $\mathrm{CO}$ and dense gas tracers seen towards the Taurus-Auriga region, and indicates that, although the bright PMS star AB Aur ( $\sim 50 L_{\odot}$, van den Ancker et al. 1998) is physically related to the cloud (Nachman 1979; Duvert et al. 1986), the bulk of the L1517 material and its embedded cores remain unperturbed by the energetic stellar output (Ladd \& Myers 1991). This combination of quiescent state, compact size, and multiple starless cores makes the L1517 cloud an ideal laboratory for studying the process of core formation. In this paper, we present a study of the physical conditions and kinematics in both the dense cores and the less-dense surrounding material using a variety of molecules known to trace different density regimes and chemical evolutionary stages of the cloud gas. As will be seen, our analysis suggests that the cores in L1517 have formed by the gravitational contraction of subsonic, velocity coherent gas in 0.5 pc-long filaments.

\section{Observations}

We observed the $\mathrm{L} 1517$ dark cloud with the FCRAO $14 \mathrm{~m}$ radio telescope during several sessions between December 2003 and November 2005. We used the 32-pixel SEQUOIA array to cover the cloud with five submaps, each of them of $10^{\prime} \times 10^{\prime}$, and observed in on-the-fly mode. The large bandpass of SEQUOIA allowed observing two different transitions simultaneously, and two passes were made to cover the cloud first in $\mathrm{N}_{2} \mathrm{H}^{+}(J=1-0)$ and $\mathrm{SO}\left(J_{\mathrm{N}}=3_{2}-2_{1}\right)$ and then in $\mathrm{C}^{18} \mathrm{O}(J=1-0)$ and $\mathrm{C}^{17} \mathrm{O}(J=$ $1-0)$. All observations were made in position-switching mode using a reference position offset by $\left(18^{\prime},-13^{\prime}\right)$ from our map center $\left(\right.$ at $\left.\alpha(\mathrm{J} 2000)=4^{\mathrm{h}} 55^{\mathrm{m}} 18^{\mathrm{s}} .8, \delta(\mathrm{J} 2000)=+30^{\circ} 38^{\prime} 04^{\prime \prime}\right)$, and known to have negligible $\mathrm{C}^{18} \mathrm{O}(J=1-0)$ emission from previous frequency-switched observations (average $\sigma\left(T_{\mathrm{mb}}\right)<0.1 \mathrm{~K}$ over the SEQUOIA footprint). The spectrometer was the DCC autocorrelator configured to provide 1024 spectral channels of $25 \mathrm{kHz}$ spacing, or approximately $0.07 \mathrm{~km} \mathrm{~s}^{-1}$ at the observing frequencies. The telescope $F W H M$ beam size varied with frequency between approximately $56^{\prime \prime}$ at the lowest $\left(\mathrm{N}_{2} \mathrm{H}^{+}\right)$frequency and $47^{\prime \prime}$ at the highest $\left(\mathrm{C}^{17} \mathrm{O}\right)$ frequency.

During the observations, calibration was achieved by measuring the emission from the sky and an ambient load every $\approx 10 \mathrm{~min}$, and the derived intensity was converted into the main beam brightness temperature scale using facility-provided main beam efficiencies close to 0.5 . The telescope pointing was checked and corrected approximately every three hours by making five-point maps on the SiO masers of IK Tau and Orion-IRc2. Typical pointing errors were within $5^{\prime \prime}$ rms.

The off-line data reduction consisted in the creation of Nyquist-sampled maps with the otftool program and a conversion to the CLASS format for further analysis with the GILDAS software (http: //www . iram. fr/IRAMFR/GILDAS). This analysis included a second-degree baseline subtraction and a spatial convolution with a Gaussian to eliminate residual noise. The final resolution of all the FCRAO data is $60^{\prime \prime}$, taking the off-line convolution into account.

Because the on-the-fly FCRAO maps are relatively shallow because of the large area covered, we complemented the $\mathrm{N}_{2} \mathrm{H}^{+}(1-0)$ FCRAO observations with a small amount of data observed with the IRAM $30 \mathrm{~m}$ radio telescope. These data were taken in frequency-switching mode and have a higher angular resolution than the FCRAO data (about 26"), but apart from that, they are consistent with the FCRAO observations in both intensity and frequency calibration. A full report of these IRAM $30 \mathrm{~m}$ data will be presented elsewhere as part of an extended study of core chemistry that includes a number of regions in addition to L1517 (Tafalla et al., in prep.). In the present paper, the IRAM $30 \mathrm{~m}$ data are only used to complement the FCRAO observations in the velocity analysis, which requires a high $S / N$ threshold.

Given the narrow lines and small velocity variations measured towards L1517, accurate rest-line frequencies were required for analyzing of the spectra. For this reason, we used the most recent laboratory estimates for $\mathrm{C}^{18} \mathrm{O}(J=1-0)$ (109782.176 MHz, see Cazzoli et al. 2003) and for $\mathrm{C}^{17} \mathrm{O}(J=$ $1-0)(112358.990 \mathrm{MHz}$ for the brightest, J F $=17 / 2-05 / 2 \mathrm{com}-$ ponent, see Cazzoli et al. 2002), which have $1 \mathrm{kHz}$ or better accuracy. For $\mathrm{N}_{2} \mathrm{H}^{+}$and $\mathrm{SO}$, no accurate laboratory measurements are available, so we used astronomical estimates. Following Pagani et al. (2009), we assumed a frequency of $93173.764 \mathrm{MHz}$ 


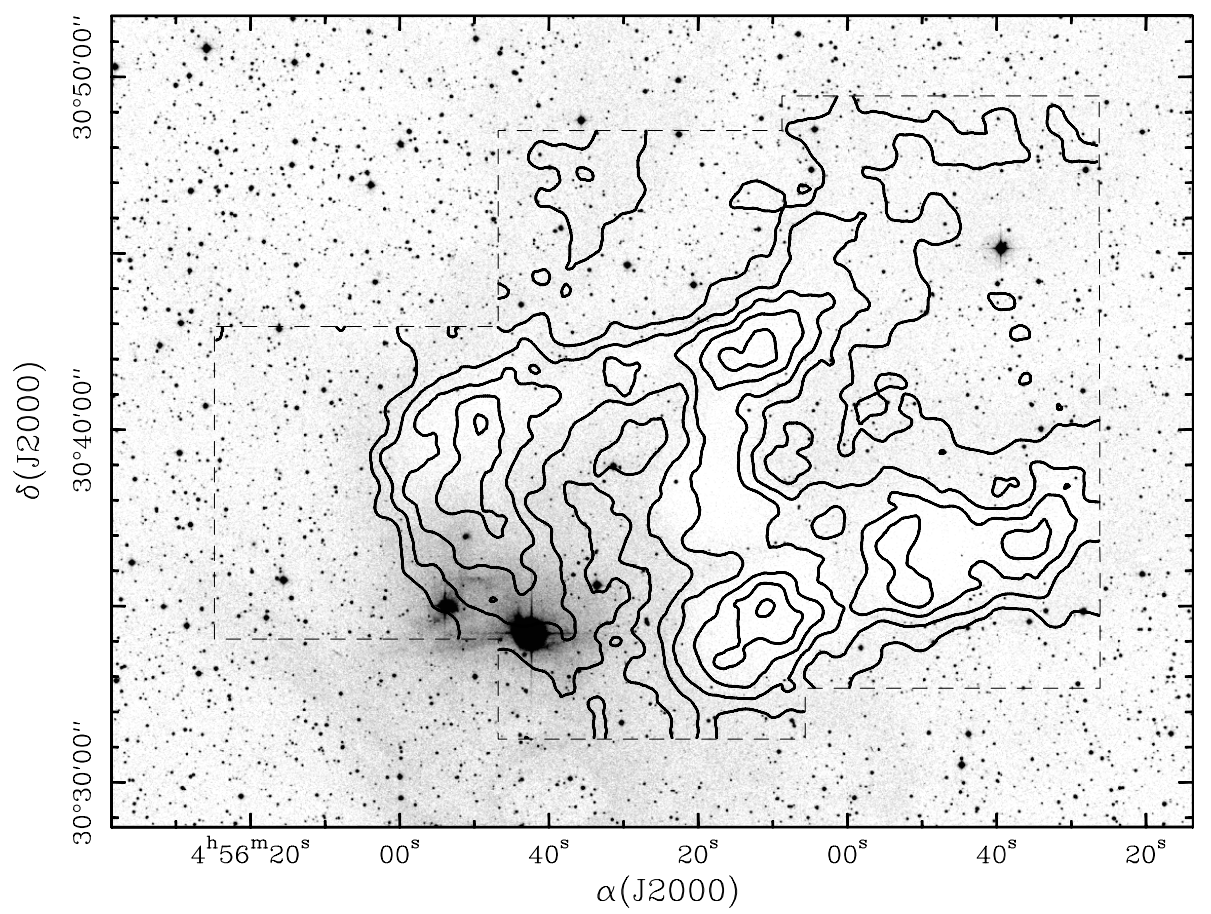

Fig. 1. Map of the $\mathrm{C}^{18} \mathrm{O}(1-0)$ integrated intensity towards the $\mathrm{L} 1517$ cloud superposed to the red DSS image. The emission has been integrated over the whole velocity range of detection $\left(V_{\mathrm{LSR}}=5.25-6.45 \mathrm{~km} \mathrm{~s}^{-1}\right)$. First contour and contour interval are $0.22 \mathrm{~K} \mathrm{~km} \mathrm{~s}^{-1}$. The two bright stars surrounded by nebulosity and located near the southeastern edge of the $\mathrm{C}^{18} \mathrm{O}(1-0)$ emission are AB Aur (brightest) and SU Aur.

for $\mathrm{N}_{2} \mathrm{H}^{+}\left(\mathrm{J} F_{1} \mathrm{~F}=123-012\right)$, with an estimated uncertainty of $4 \mathrm{kHz}$, and following Tafalla et al. (2006), we assumed a frequency of $99299.890 \mathrm{MHz}$ for $\mathrm{SO}\left(J_{\mathrm{N}}=3_{2}-2_{1}\right)$, with an estimated uncertainty of $10 \mathrm{kHz}$.

Additional observations of the dense cores in L1517 were carried out in the $1.2 \mathrm{~mm}$ continuum using the MAMBO array on the IRAM $30 \mathrm{~m}$ radio telescope during several sessions between December 1999 and January 2005. The data for core L1517B have already been presented in Tafalla et al. (2004), while the data for cores L1517A and L1517C are newly reported here. (L1517D was not observed.) In all cases, the observations were done in on-the-fly mode scanning the telescope in azimuth at a speed of $4^{\prime \prime} \mathrm{s}^{-1}$, and using wobbler switching with a period of $0.5 \mathrm{~s}$ and a throw of $53^{\prime \prime}$ or $70^{\prime \prime}$. The raw data were corrected for atmospheric attenuation using sky dips usually made before and/or after the source observation, and the absolute intensity calibration was achieved using facility-provided factors derived from observations of planets. The older L1517B data were reduced with the NIC software using no method of noise reduction (to avoid filtering the extended emission), while the newer L1517A and L1517C data were reduced with MOPSIC using a noise reduction method optimized to recover the extended emission. The intrinsic beam size of all the continuum data is $11^{\prime \prime}$, although the maps were later convolved to an equivalent resolution of $20^{\prime \prime}$ to eliminate high spatial-frequency noise.

\section{3. $\mathrm{C}^{18} \mathrm{O}$ data: the extended cloud}

Figure 1 presents our map of $\mathrm{C}^{18} \mathrm{O}(1-0)$ emission integrated over the full velocity range of detection superposed to the red DSS image. This map is in good agreement with the previous map by Heyer et al. (1987), and it shows how the $\mathrm{C}^{18} \mathrm{O}(1-0)$ emission extends to the northwest of AB and SU Aur, the brightest stars in the DSS image. These PMS stars are physically associated with the L1517 cloud, as indicated by the heating of the gas in their vicinity (Nachman 1979; Duvert et al. 1986), but their effect must be highly localized since the data described in the next sections show no evidence of any kinematic interaction between the gas and the stars. This lack of kinematical interaction, together with the fact that the northwest elongation of the L1517 cloud continues in the maps of Duvert et al. (1986) to scales as large as $4 \mathrm{pc}$ (or more than 5 times the size of our map) and involves additional dark clouds such as L1496, L1505, L1513, and L1515, suggests that the distribution of gas in L1517 is intrinsic to the cloud, and that it has not been sculpted by the PMS stars. In this sense, the L1517 cloud seems a quiescent remnant of a larger gas cloud that gave rise to AB Aur, SU Aur, and an additional group of PMS stars located in their vicinity (Luhman et al. 2009).

\subsection{Filament identification}

A more detailed view of the gas distribution in L1517 comes from the analysis of its velocity structure. Figure 2 shows maps of $\mathrm{C}^{18} \mathrm{O}(1-0)$ emission integrated every $0.4 \mathrm{~km} \mathrm{~s}^{-1}$, or about twice the sound speed for gas at $10 \mathrm{~K}$. These maps, and others made using different velocity ranges, show that the L1517 cloud is structured in at least four elongated components that are referred to as filaments 1 to 4 . These four filaments can also be seen in maps of extinction (Jouni Kainulainen, priv. comm.), ${ }^{13} \mathrm{CO}$ (Heyer et al. 1987), and SO emission (Sect. 4), so they must reflect the true distribution of gas in the L1517 cloud, and are not mere artifacts of the $\mathrm{C}^{18} \mathrm{O}$ chemistry or excitation.

As Fig. 2 shows, each filament appears in only one $0.4 \mathrm{~km} \mathrm{~s}^{-1}$-wide channel map, indicating that its gas is highly confined in velocity space. This low level of velocity structure results from a very quiescent state of the gas in the filaments, both in terms of velocity dispersion and internal velocity gradients (to be discussed below), and makes it possible to associate each element of emission in the cloud to one of the four 


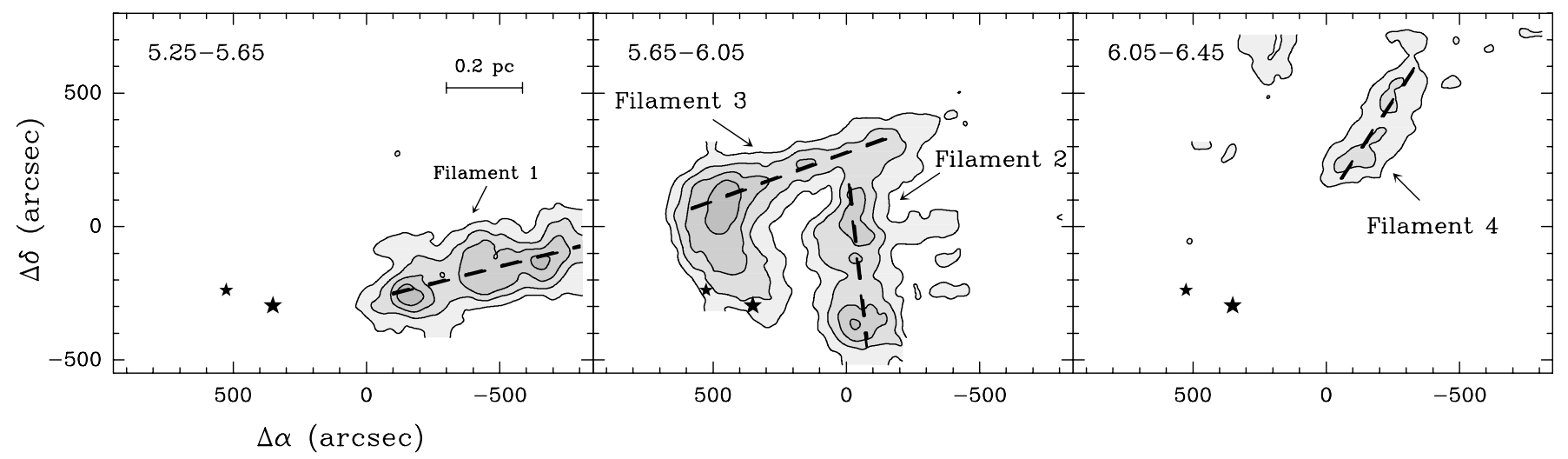

Fig. 2. Maps of $\mathrm{C}^{18} \mathrm{O}(1-0)$ intensity integrated every $0.4 \mathrm{~km} \mathrm{~s}^{-1}$ to illustrate the separation of the gas into filaments. The dashed lines indicate our best guess of each filament axis, and the star symbols mark the positions of AB Aur (larger) and SU Aur (smaller). First contour and interval are $0.2 \mathrm{~K} \mathrm{~km} \mathrm{~s}^{-1}$. Offsets are referred to $\alpha(\mathrm{J} 2000)=4^{\mathrm{h}} 55^{\mathrm{m}} 18.8, \delta(\mathrm{J} 2000)=+30^{\circ} 38^{\prime} 04^{\prime \prime}$. The LSR velocity range of integration is indicated in the upper left corner of each panel.

Table 1. Filaments in L1517.

\begin{tabular}{lcccc}
\hline \hline Filament & $\begin{array}{c}V_{\text {LSR range }} \\
\left(\mathrm{km} \mathrm{s}^{-1}\right)\end{array}$ & $\begin{array}{c}\text { Length }^{1} \\
(\mathrm{pc})\end{array}$ & $\begin{array}{c}\text { Mass }^{2} \\
\left(M_{\odot}\right)\end{array}$ & Cores \\
\hline 1 & {$[5.25-5.65]$} & 0.52 & 8.0 & $\mathrm{~A} 2, \mathrm{C}$ \\
2 & {$[5.65-6.05]$} & 0.42 & 7.2 & $\mathrm{~A} 1, \mathrm{~B}$ \\
3 & {$[5.65-6.05]$} & 0.70 & 11.3 & $\mathrm{D}$ \\
4 & {$[6.05-6.45]$} & 0.38 & 4.8 & - \\
\hline
\end{tabular}

Notes. ${ }^{(1)}$ Uncorrected for projection effects; ${ }^{(2)}$ from $\mathrm{C}^{18} \mathrm{O}(1-0)$ emission.

filaments. To carry out this assignment, we have taken both the spatial location of the emission and its velocity into account and assumed that the velocity limits of the filaments are those used in the maps of Fig. 2 (see also Table 1). This assigning procedure works well over most of the mapped region, but it provides ambiguous answers in the vicinity of offsets $\left(-100^{\prime \prime},-300^{\prime \prime}\right)$. In this region, filaments 1 and 2 overlap spatially and converge in velocity to a value close to $5.65 \mathrm{~km} \mathrm{~s}^{-1}$, which is our assumed boundary between the two filaments. A detailed inspection of the FCRAO spectra in this region (supplemented with additional data from the IRAM 30 m telescope) reveals that the emission from the two filaments becomes almost indistinguishable, either because the filaments merge physically or because they superpose along the line of sight with similar kinematic properties. As a result, assigning emission in this region to either filament 1 or 2 becomes more uncertain than in other places of the cloud. Although this may lead to some confusion in a localized region, it is unlikely to affect our global analysis of the gas kinematics, since the properties of the two filaments become so similar in terms of velocity centroid and linewidth that an erroneous assignment of a gas parcel to either filament will only add a small amount of mass, but will not change its velocity field.

Once we have decomposed the L1517 cloud into its constituent filaments, we use the $\mathrm{C}^{18} \mathrm{O}(1-0)$ emission to estimate their basic physical parameters, and we summarize the results in Table 1. From the maps of Fig 2, we estimate that the filaments have lengths of 550-1000 arcsec, which correspond to physical sizes of $\sim 0.35-0.70 \mathrm{pc}$ for our adopted distance of $144 \mathrm{pc}$ (based on the Hipparcos distance to AB Aur, van den Ancker et al. 1998). These sizes represent a significant fraction of the total cloud length, and this reinforces the idea that the cloud is structured as a network of filaments. To estimate the masses of the filaments, we use the $\mathrm{C}^{18} \mathrm{O}(1-0)$ emission and assume that it is optically thin and in LTE at $10 \mathrm{~K}$. For a $\mathrm{C}^{18} \mathrm{O}$ abundance of $1.7 \times 10^{-7}$ (Frerking et al. 1982), the typical filament masses are $5-11 M_{\odot}$, which imply linear mass densities in the range 12 $17 M_{\odot} \mathrm{pc}^{-1}$.

\subsection{Density structure of the filaments}

As Fig. 2 shows, the $\mathrm{C}^{18} \mathrm{O}$ emission from the filaments displays a significant degree of central brightening. This suggests that the underlying density structure of the filaments is centrally concentrated and that the $\mathrm{C}^{18} \mathrm{O}$ emission can be used to determine each filament density profile. To carry out this determination, we first defined a central axis for each filament assuming that they are rectilinear and show the results in Fig. 2. As can be seen, the rectilinear approximation is reasonable for all filaments but number 3, which bends towards the location of the PMS stars near its southeast end. Whether this bend is related to core L1517D, with which it coincides in position (Sect. 4), or is associated to the PMS stars is unclear from our data. In either case, for simplicity in the modeling, we approximate all filaments by straight lines, with the caveat that this approach is only a first-order approximation.

Using the above linear axes, we created a profile of $\mathrm{C}^{18} \mathrm{O}(1-$ $0)$ intensity for each filament as a function of cylindrical radius. The result, presented in Fig. 3, shows that the four filaments in the cloud have centrally concentrated distributions with a flattened region close to the axis and an approximate powerlaw tail at large distances. Filament 3 presents the highest dispersion of the sample due to a combination of contamination from filament 2 emission near its northern edge and the additional contribution from the "bend" region previously discussed. Nevertheless, given the rather irregular distribution of emission seen in Fig. 2, it is remarkable that the radial profile of each filament presents relatively little scatter and that all the filaments seem to follow a very similar type of radial profile.

The regular behavior of the radial profiles in Fig. 3 suggests that the underlying density structure of the filaments can be described with a simple density law. To derive such a law, we model the $\mathrm{C}^{18} \mathrm{O}(1-0)$ emission assuming that each filament has cylindrical symmetry and that the $\mathrm{C}^{18} \mathrm{O}$ emission arises from gas in LTE thermalized at $10 \mathrm{~K}$, as suggested by the study of the L1517B core and its surrounding envelope in Tafalla et al. (2004). Under these conditions, if we assume a density profile, together with a constant $\mathrm{C}^{18} \mathrm{O}$ abundance $\left(1.7 \times 10^{-7}\right.$, Frerking et al. 1982) and a $\mathrm{C}^{18} \mathrm{O}$ linewidth of $0.3 \mathrm{~km} \mathrm{~s}^{-1}$ (Sect. 6.1), we 
A. Hacar and M. Tafalla: Fragmentation of velocity coherent filaments in L1517

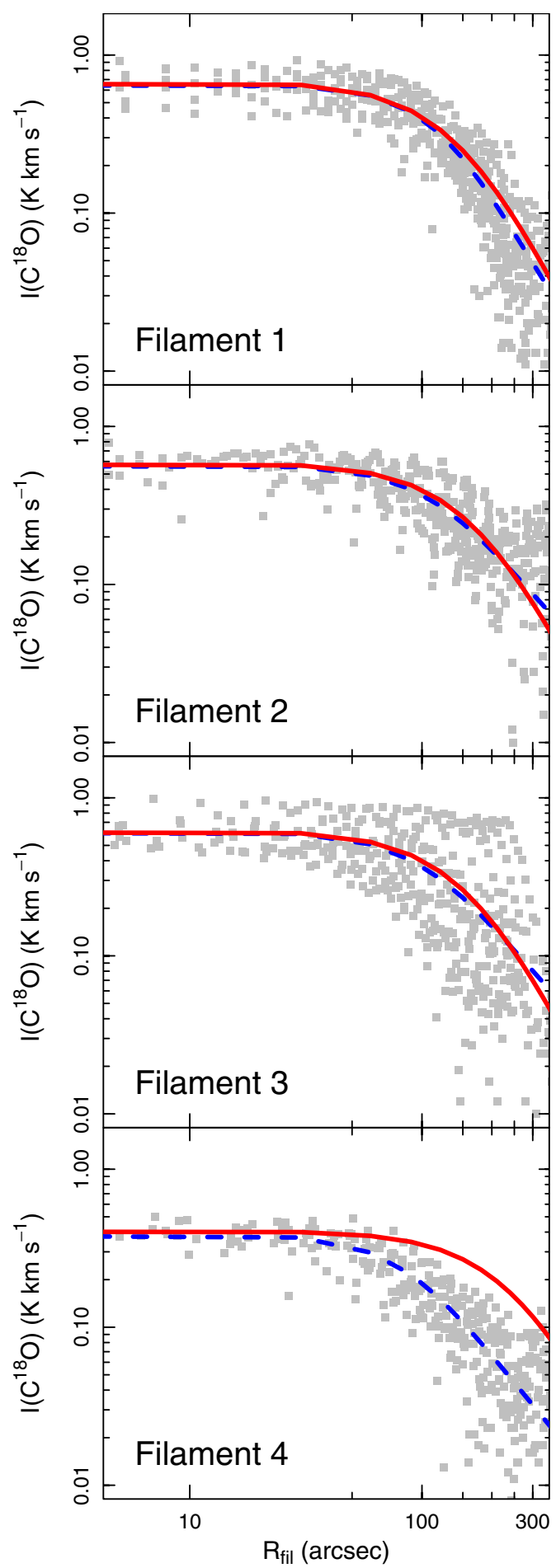

Fig. 3. $\mathrm{C}^{18} \mathrm{O}(1-0)$ emission profiles across each of the $\mathrm{L} 1517$ filaments. The points represent the integrated emission within the velocity range of each filament (see Table 1), and $R_{\text {fil }}$ is the distance to the filament axis. For each filament, the red solid curve indicates the isothermal cylinder model that fits the emission towards the axis, and the blue dashed line is the best-fit softened power law.

can predict a radial profile of emission that can be compared, after appropriate beam convolution, with the observed radial profiles shown in Fig. 3.

As a first guess for the filament density profiles, we use the family of isothermal cylinders in pressure equilibrium with their self gravity. This family was originally described by
Table 2. Fits to the $\mathrm{C}^{18} \mathrm{O}$ radial profiles ${ }^{1}$.

\begin{tabular}{c|cc|ccc}
\hline \hline \multirow{3}{*}{ Filament } & \multicolumn{2}{|c|}{ Isothermal cylinder } & \multicolumn{3}{|c}{ Softened power law } \\
& $n_{\circ}$ & $r_{1 / 2}^{2}$ \\
$\left(\mathrm{~cm}^{-3}\right)$ & $(\operatorname{arcsec})$ & $n_{\circ}$ & $\begin{array}{c}r_{1 / 2} \\
\left(\mathrm{~cm}^{-3}\right)\end{array}$ & $\alpha$ \\
& $1 \times 10^{4}$ & 89 & $8.5 \times 10^{3}$ & 105 & 3.4 \\
\hline 1 & $7 \times 10^{3}$ & 106 & $7 \times 10^{3}$ & 100 & 2.7 \\
3 & $8 \times 10^{3}$ & 99 & $8.5 \times 10^{3}$ & 90 & 2.7 \\
4 & $3 \times 10^{3}$ & 162 & $6.5 \times 10^{3}$ & 75 & 2.8 \\
\hline
\end{tabular}

Notes. ${ }^{(1)}$ Not corrected for inclination; ${ }^{(2)} r_{1 / 2}=\left(2^{1 / 2}-1\right)^{1 / 2} H$.

Stodólkiewicz (1963) and Ostriker (1964), and has the simple analytic form

$n(r)=\frac{n_{\circ}}{\left(1+(r / H)^{2}\right)^{2}}$,

where $r$ is the cylindrical radius, $n_{\circ}$ is the central density,

$H^{2}=\frac{2 c_{\mathrm{s}}^{2}}{\pi G \mu n_{\circ}}$

$c_{\mathrm{S}}$ is the isothermal sound speed, $G$ the gravitational constant, and $\mu$ the mean molecular mass. For the case of L1517, the gas kinetic temperature is known to be approximately $10 \mathrm{~K}$ (Tafalla et al. 2004) so any isothermal cylinder is described by just one parameter, its central density $n_{\circ}$. This means that once we have set the central density to a value that fits the intensity at zero radius, we do not have any free parameter left to control the rest of the radial profile, and in particular, the width at half maximum of the emission is automatically determined by the $H$ value.

There are a number of motivations for using the isothermal cylinder family as a first choice to fit the $\mathrm{C}^{18} \mathrm{O}$ radial profiles. In addition to the inherent simplicity and assumption of equilibrium of the model, the isothermal cylinder is attractive for presenting both a central density flattening and a power-law tail, which are two characteristics of the observed radial profiles. The isothermal cylinder, in addition, has a mass per unit length that is independent of the central density and is only a function of the gas temperature (Ostriker 1964). For the $10 \mathrm{~K}$ assumed for the gas in L1517, this mass per unit length is approximately $16 M_{\odot} \mathrm{pc}^{-1}$, which is reasonably close to the mass per unit length of the filaments estimated in Table 1.

The results of our isothermal cylinder fits to the filament profiles in L1517 are shown in Fig. 3. These fits were selected to match the $\mathrm{C}^{18} \mathrm{O}$ emission towards the filament axis, so the radius of half maximum emission is a direct prediction from the model so is not controlled by our fit. As can be seen, the predicted filament widths are in reasonable agreement with the observations, with only filament 4 being clearly narrower than predicted by the model. In addition, the central densities predicted by the fits range from $3 \times 10^{3}$ to $1 \times 10^{4} \mathrm{~cm}^{-3}$ (see Table 2), and these values agree with previous estimates of the gas density in the extended part of the L1517 cloud (Tafalla et al. 2004).

To check the consistency of the parameters derived with the isothermal cylinder model and to provide a better match to the radial profiles of the filaments, we have fitted the $\mathrm{C}^{18} \mathrm{O}$ emission with an alternative family of profiles that also have central flattening and asymptotic power-law behavior. This family consists of softened power laws described by

$n(r)=\frac{n_{\circ}}{1+\left(r / r_{1 / 2}\right)^{\alpha}}$, 
where $n_{\circ}$ is again the central density, $r_{1 / 2}$ the half-density radius, and $\alpha$ the asymptotic power index. This family of profiles has been shown to fit the density structure of starless cores, which also present flattened central regions and asymptotic power-law behavior (Tafalla et al. 2004). In contrast to the equilibrium cylinder, the softened power law has three free parameters, so it fits the central density and the width of the filament independently. By comparing the results from the softened power law fits with those from the isothermal cylinders, we can now test how self consistent the one-parameter isothermal-cylinder fits are.

Figure 3 shows the softened power law fits derived with a chi-squared minimization algorithm. As can be seen, these fits are almost indistinguishable from the isothermal cylinder fits for filaments 1,2 , and 3, and the only clear difference between the two families of fits occurs in filament 4. A more quantitative comparison between the fits comes from examining the derived central density and filament width in the two families. Table 2 shows that these parameters differ by less than $10 \%$ on average for the first three filaments, indicating again that the isothermal cylinder and softened power-law fits are equivalent within the scatter of the observations. In filament 4 , the softened powerlaw fit is clearly superior to the (poor) isothermal cylinder fit, and the difference between the derived parameters in the density law is approximately a factor of 2 .

\subsection{Implications and limitations of the modeling}

Our analysis of the L1517 filaments adds to a number of previous studies of filamentary structures. With few exceptions, like that of Johnstone et al. (2003) who fitted the $850 \mu \mathrm{m}$ emission from the infrared-dark cloud G11.11-0.12, most previous work has found significant deviations between the radial profiles of the filaments and the prediction from the isothermal cylinder model, usually because the observed asymptotic power-law behavior is flatter than the $r^{-4}$ predicted by the isothermal cylinder model (Alves et al. 1998; Stepnik et al. 2003; André et al. 2010; Arzoumanian et al. 2011). For the filaments in L1517, our radial profiles do not extend far enough in radius to sample the $r^{-4}$ asymptotic behavior, as can be seen in Fig. 3 from the isothermal cylinder models being practically indistinguishable from softened power-law profiles with an asymptotic behavior close to $r^{-2.7}$ (Table 2). As a result, testing whether the L1517 filaments follow the prediction from the model or deviate from it cannot be done with our data, and it requires extending the radial profiles to larger radii, preferably using more robust techniques, such as extinction measurements or dust continuum observations.

Even if the L1517 filaments do not follow the expected asymptotic behavior, it is striking that they approximately fit the expectations from the isothermal cylinder model both in width and mass per unit length. Clearly there are a number of limitations in our study, like the use of the freeze-out prone $\mathrm{C}^{18} \mathrm{O}$ molecule as a column density tracer and our ignoring of projection effects, so there is probably room for a factor of 2 uncertainty in the results. Also, as we will see in Sect. 8, the size scale of fragmentation suggests that there may be a contribution from additional forces, like external pressure or magnetic fields (which could even flatten the radial profile, e.g., Fiege \& Pudritz 2000a). Still, the very quiescent state of the cloud described in the following sections indicates that the gas in L1517 cannot be too far from a state of equilibrium, as otherwise it would quickly develop supersonic motions, (e.g., Burkert \& Hartmann 2004) and would not fragment into well-separated cores (Inutsuka \& Miyama 1997). As pressure forces dominate, it seems reasonable to expect that the distribution of mass in the filaments bears some similarity to the prediction from the isothermal cylinder model.

\section{Dense core population}

The population of dense cores in the L1517 cloud was first described by Schneider \& Elmegreen (1979), who identified four regions of enhanced obscuration in the Palomar optical plates and named them A to D in order of increasing right ascension (RA). For unknown reasons, this original labeling scheme was later replaced by one in which the cores are named C, A, B, and D in order of increasing RA, and this notation has been used by most core studies in the past (e.g., Benson \& Myers 1989). For consistency with previous work, we also use this disordered core labeling here.

Figure 4 presents different views of the core population in L1517. The optical DSS image on the top left has had its contrast enhanced to better show the cores as dark patches against both the galactic stellar background and the bright diffuse emission from AB Aur and SU Aur, which are probably slightly behind the cores. The other panels show maps of the same region in the $1.2 \mathrm{~mm}$ dust continuum, $\mathrm{N}_{2} \mathrm{H}^{+}(1-0)$, and $\mathrm{SO}\left(3_{2}-2_{1}\right)$, three tracers that highlight different properties of the dense, high column density gas. The $1.2 \mathrm{~mm}$ continuum emission is mostly sensitive to the column density of the cores (the extended emission from the filament is filtered out by the bolometer), and its maps provide high angular resolution views of cores A, B, and C (core D was not mapped). The brightest $1.2 \mathrm{~mm}$ emission corresponds to core $\mathrm{B}$, whose structure and chemical composition has been studied in detail by Tafalla et al. (2004, 2006). Cores A and $\mathrm{C}$ have received less attention due to their weaker emission in both high-density tracers and mm-continuum (Benson \& Myers 1989; Ladd \& Myers 1991; Kirk et al. 2005), so their structure is less well known. Our mm-continuum map shows that core A is double-peaked, while core $\mathrm{C}$ is single-peaked but relatively more extended and significantly weaker than cores A and B. Overall, the mm-continuum map is characterized by a lack of point-like components, which together with the absence of Spitzer $24 \mu \mathrm{m}$ point sources (Kirk et al. 2007b) is a strong indication that all dense cores in the cloud are starless.

The $\mathrm{N}_{2} \mathrm{H}^{+}$and $\mathrm{SO}$ maps in Fig. 4 present complementary views of the $\mathrm{L} 1517$ cores. $\mathrm{N}_{2} \mathrm{H}^{+}$is a so-called latetime molecule, and its abundance is further enhanced when $\mathrm{CO}$ freezes out (e.g., Bergin \& Tafalla 2007), so bright $\mathrm{N}_{2} \mathrm{H}^{+}$ emission is commonly associated with evolved cores (Crapsi et al. 2005). As Fig. 4 shows, the $\mathrm{N}_{2} \mathrm{H}^{+}$emission is brightest towards cores $\mathrm{B}$ and $\mathrm{D}$, and still noticeable towards core A1. These cores therefore seem more chemically evolved than cores $\mathrm{C}$ and $\mathrm{A} 2$, which are barely detected in $\mathrm{N}_{2} \mathrm{H}^{+}(1-0)$. Such interpretation of the $\mathrm{N}_{2} \mathrm{H}^{+}$maps in terms of chemical evolution is supported by the distribution of SO emission. Observationally, SO is known to be highly sensitive to molecular freeze out (Tafalla et al. 2006), and in addition, it is predicted to decrease in abundance with time (Bergin \& Langer 1997; Aikawa et al. 2005). The SO emission, therefore, is expected to be anticorrelated with that of $\mathrm{N}_{2} \mathrm{H}^{+}$, and indeed, the maps of $\mathrm{L} 1517$ show such behavior: the $\mathrm{SO}$ emission is dominated by cores $\mathrm{C}$ and $\mathrm{A} 2$, which are weak in $\mathrm{N}_{2} \mathrm{H}^{+}(1-0)$, while the $\mathrm{N}_{2} \mathrm{H}^{+}$-bright cores $\mathrm{A} 1$, B, and $\mathrm{D}$ are barely distinguishable from the $\mathrm{SO}$ emission of the extended cloud.

\subsection{Core emission modeling}

To quantify the physical and chemical properties of the L1517 cores, we modeled their emission following the procedure 
A. Hacar and M. Tafalla: Fragmentation of velocity coherent filaments in L1517

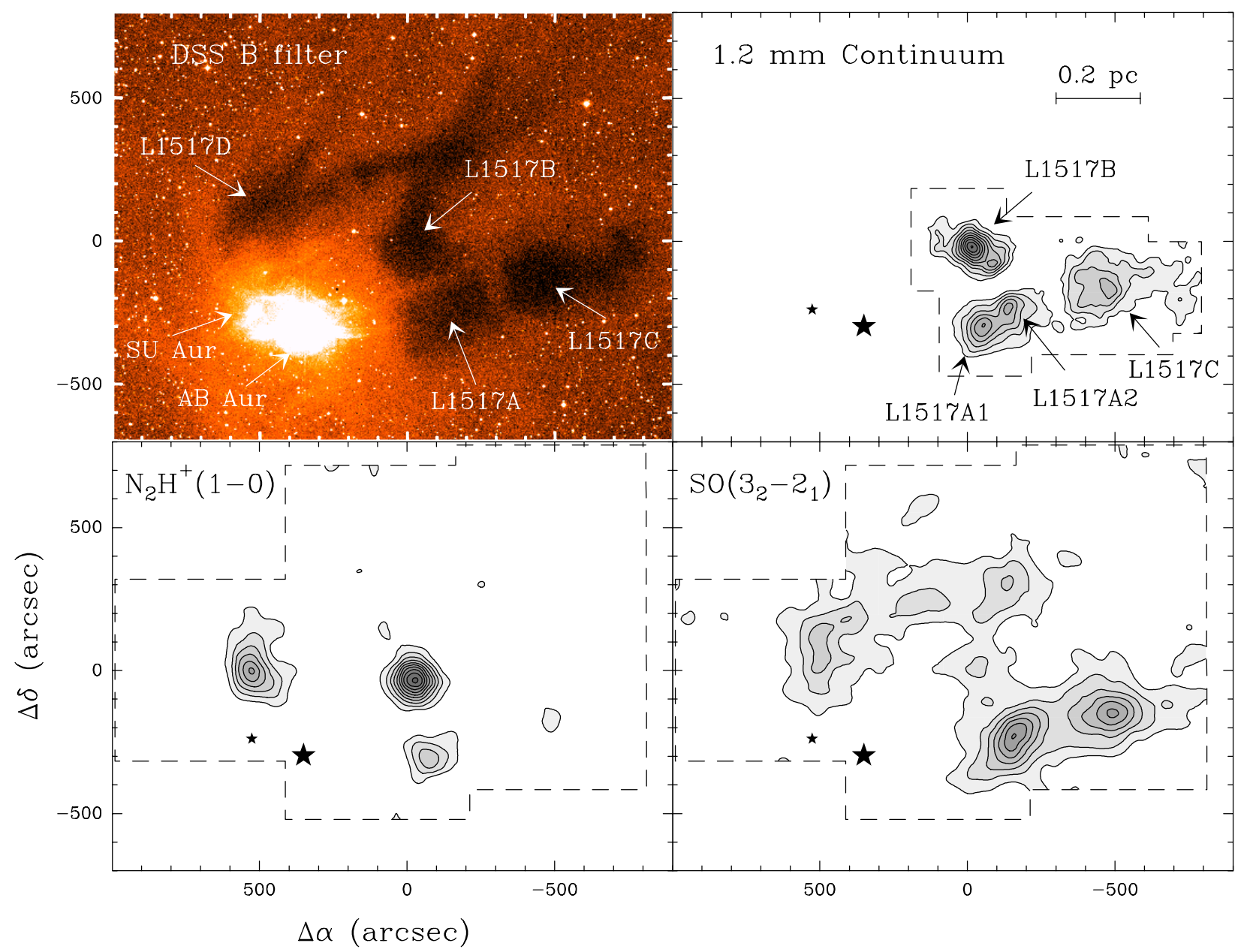

Fig. 4. Core population of L1517. From left to right and from top to bottom: contrast-enhanced DSS blue image identifying the core positions, $1.2 \mathrm{~mm}$ dust continuum emission, $\mathrm{N}_{2} \mathrm{H}^{+}(1-0)$ integrated intensity, and $\mathrm{SO}\left(3_{2}-2_{1}\right)$ integrated intensity. Offsets and star symbols are as in Fig. 2. First contour and spacing are $2 \mathrm{mJy} / 11^{\prime \prime}$-beam for $1.2 \mathrm{~mm}$ continuum, $0.16 \mathrm{~K} \mathrm{~km} \mathrm{~s}^{-1}$ for $\mathrm{N}_{2} \mathrm{H}^{+}(1-0)$, and $0.08 \mathrm{~K} \mathrm{~km} \mathrm{~s}^{-1}$ for $\mathrm{SO}\left(3_{2}-2_{1}\right)$. To enhance the sensitivity to extended emission, the $1.2 \mathrm{~mm}$ continuum map has been convolved to an equivalent resolution of $30^{\prime \prime}$, and the $\mathrm{N}_{2} \mathrm{H}^{+}$and $\mathrm{SO}$ maps to a resolution of $75^{\prime \prime}$.

described in Tafalla et al. (2004) for the analysis of the L1498 and L1517B cores. In this way, we assumed that the cores are spherically symmetric, and we concentrated our modeling effort on fitting the radial profiles of emission shown in Fig. 5. To determine the density profile of each core, we fitted the $1.2 \mathrm{~mm}$ continuum emission, as this is expected to be the most faithful tracer of the total dust and gas column densities (e.g., Bergin \& Tafalla 2007). Following the analysis of L1517B, we assumed a uniform dust temperature of $T_{\mathrm{d}}=10 \mathrm{~K}$ and a $1.2 \mathrm{~mm}$ emissivity of $\kappa=0.005 \mathrm{~cm}^{2} \mathrm{~g}^{-1}$, and we fitted the continuum radial profiles with density laws of the form $n(r)=n_{0} /\left(1+\left(r / r_{0}\right)^{\alpha}\right)$, where $n_{0}$, $r_{0}$, and $\alpha$ are free parameters. (For core $\mathrm{B}$, we have used the Tafalla et al. 2004 result, while core D was not fitted for lack of continuum data.) The results of these fits are illustrated in Fig. 5, and the best-fit parameters are summarized in Table 4 . As can be seen, core central densities range from $4.7 \times 10^{4} \mathrm{~cm}^{-3}$ in core $\mathrm{C}$ to $2.2 \times 10^{5} \mathrm{~cm}^{-3}$ in core $\mathrm{B}$, which correspond, respectively, to enhancements of 6 and 30 with respect to the central density of the filaments traced in $\mathrm{C}^{18} \mathrm{O}$ (Sect. 3.2). Integrating the density profiles up to a representative radius of $0.05 \mathrm{pc}\left(\approx 75^{\prime \prime}\right)$, we estimate that the core masses are on the order of $1-2 M_{\odot}$, which is typical of the population of Taurus starless cores (Benson \& Myers 1989).

Once the density profile of each core had been modeled, we could determine the abundance of the different species by
Table 3. Kinematic properties of the L1517 cores.

\begin{tabular}{lcccc}
\hline \hline Core & $\begin{array}{c}\Delta \alpha, \Delta \delta \\
\left({ }^{\prime \prime},{ }^{\prime \prime}\right)\end{array}$ & $\begin{array}{c}V_{\text {lsr }}\left(\mathrm{N}_{2} \mathrm{H}^{+}\right) \\
\left(\mathrm{km} \mathrm{s}^{-1}\right)\end{array}$ & $\begin{array}{c}\Delta V\left(\mathrm{~N}_{2} \mathrm{H}^{+}\right) \\
\left(\mathrm{km} \mathrm{s}^{-1}\right)\end{array}$ & $\begin{array}{c}\left\langle\left|\nabla V_{\text {lsr }}\left(\mathrm{N}_{2} \mathrm{H}^{+}\right)\right|\right\rangle \\
\left(\mathrm{km} \mathrm{s}^{-1} \mathrm{pc}^{-1}\right)\end{array}$ \\
\hline $\mathrm{A} 1$ & $-60,-300$ & 5.70 & 0.18 & $0.7 \pm 0.1$ \\
$\mathrm{~A} 2^{1}$ & $-150,-240$ & 5.57 & 0.20 & $-{ }^{2}$ \\
$\mathrm{~B}$ & $-30,-30$ & 5.79 & 0.22 & $0.7 \pm 0.3$ \\
$\mathrm{C}^{1}$ & $-480,-150$ & 5.48 & 0.18 & $-^{2}$ \\
$\mathrm{D}$ & 540,0 & 5.88 & 0.29 & $0.9 \pm 0.4$ \\
\hline
\end{tabular}

Notes. ${ }^{(1)}$ Kinematics data from IRAM 30 m observations (rest from FCRAO $14 \mathrm{~m}$ ). ${ }^{(2)}$ Not enough data to estimate gradient.

solving the equation of radiative transfer and convolving the result with the appropriate Gaussian beam size to simulate an observation. We did this using a Monte Carlo non-LTE code based on that of Bernes (1979), and we excluded core D from the analysis due to the lack of mm-continuum data. For all the cores, we assumed a gas kinetic temperature of $9.5 \mathrm{~K}$ based on the $\mathrm{NH}_{3}$ analysis of L1517B in Tafalla et al. (2004), and in agreement with typical estimates for other low-mass starless cores (Benson \& Myers 1989). To reproduce the typical $\mathrm{N}_{2} \mathrm{H}^{+}(1-0) F W H M$ of $0.21 \mathrm{~km} \mathrm{~s}^{-1}$ (mean of values for all cores, see Table 3 ), we added a constant nonthermal component of $0.17 \mathrm{~km} \mathrm{~s}^{-1}(F W H M)$ to the 


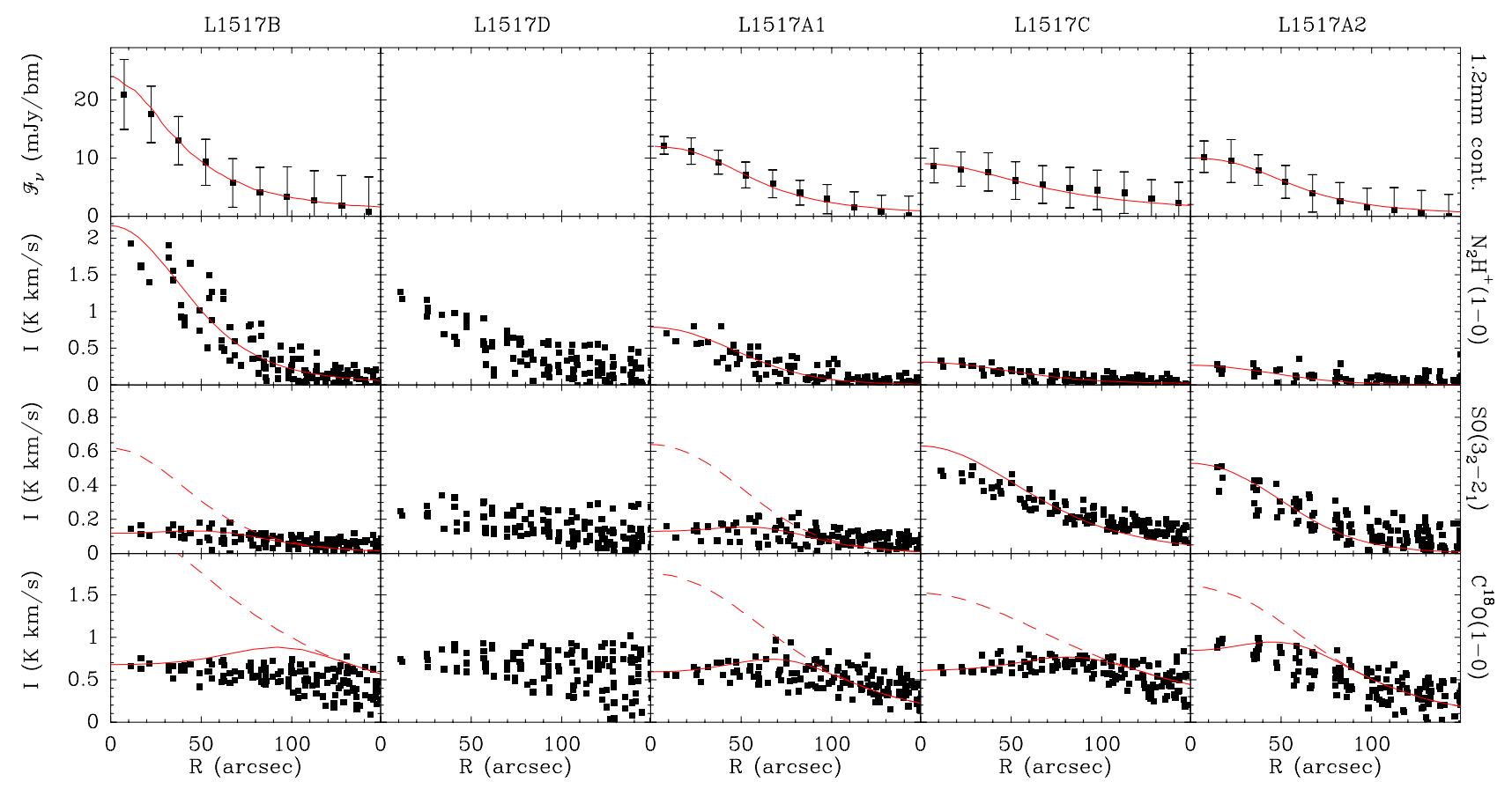

Fig. 5. Radial profiles of $1.2 \mathrm{~mm}$ continuum, $\mathrm{N}_{2} \mathrm{H}^{+}(1-0), \mathrm{SO}\left(3_{2}-2_{1}\right)$, and $\mathrm{C}^{18} \mathrm{O}(1-0)$ emission towards the dense cores in $\mathrm{L} 1517$. The cores were ordered by decreasing central emission of $\mathrm{N}_{2} \mathrm{H}^{+}(1-0)$ to illustrate a possible evolutionary sequence (see text). The $1.2 \mathrm{~mm}$ data were averaged over $15^{\prime \prime}$-wide intervals to increase legibility. In each panel, the solid red line represents the best fit-model, and when an additional dashed line is presented, the best fit required a central abundance hole and the dashed line represents the constant abundance model for comparison. No modeling of core $\mathrm{D}$ was attempted for lack of mm-continuum data.

Table 4. Density and abundance profiles in the L1517 cores $^{1}$.

\begin{tabular}{l|ccc|ccccc}
\hline \hline & \multicolumn{3}{|c|}{ Density parameters } & \multicolumn{4}{c}{ Molecular abundances and depletion radii } \\
Core & $\begin{array}{c}n_{\circ} \\
\left(\mathrm{cm}^{-3}\right)\end{array}$ & $\begin{array}{c}r_{1 / 2} \\
(\operatorname{arcsec})\end{array}$ & $\alpha$ & $\mathrm{X}\left(\mathrm{N}_{2} \mathrm{H}^{+}\right)$ & $\mathrm{X}(\mathrm{SO})$ & $r_{\mathrm{d}}(\mathrm{SO})$ & $\mathrm{X}\left(\mathrm{C}^{18} \mathrm{O}\right)$ & $r_{\mathrm{d}}\left(\mathrm{C}^{18} \mathrm{O}\right)$ \\
& & & & $(\mathrm{cm})$ & & $(\mathrm{cm})$ \\
\hline $\mathrm{A} 1$ & $7.0 \times 10^{4}$ & 60 & 2.5 & $1.0 \times 10^{-10}$ & $1.0 \times 10^{-9}$ & $1.3 \times 10^{17}$ & $1.5 \times 10^{-7}$ & $1.5 \times 10^{17}$ \\
$\mathrm{~A} 2$ & $6.0 \times 10^{4}$ & 60 & 3.5 & $0.4 \times 10^{-10}$ & $1.0 \times 10^{-9}$ & 0.0 & $1.5 \times 10^{-7}$ & $1.1 \times 10^{17}$ \\
$\mathrm{~B}$ & $2.2 \times 10^{5}$ & 35 & 3.5 & $1.5 \times 10^{-10}$ & $0.4 \times 10^{-9}$ & $1.2 \times 10^{17}$ & $1.5 \times 10^{-7}$ & $1.9 \times 10^{17}$ \\
$\mathrm{C}$ & $4.7 \times 10^{4}$ & 60 & 2.5 & $0.7 \times 10^{-10}$ & $2.0 \times 10^{-9}$ & 0.0 & $1.5 \times 10^{-7}$ & $1.7 \times 10^{17}$ \\
\hline
\end{tabular}

Notes. ${ }^{(1)}$ All abundances are relative to $\mathrm{H}_{2}$. Core D was not modeled due to lack of mm-continuum data.

velocity field. Also, we extended each core density profile with a cloud component based on the profile of the filament in which it is embedded. The assignment of cores to filaments was done using the core LSR velocity determined from $\mathrm{N}_{2} \mathrm{H}^{+}$(Table 3 ) and the velocity limits of each filament measured from $\mathrm{C}^{18} \mathrm{O}$ (Table 1). According to this criterion, cores $\mathrm{A} 2$ and $\mathrm{C}$ belong to filament 1 , cores A1 and B to filament 2, and core D to filament 3 (as summarized in Table 1). As discussed in Sect. 3.1, there is some ambiguity in the assignment of gas to filaments in the region where filaments 1 and 2 overlap, and this may affect the filament assignment for cores A1 and A2. Our choice denotes our best understanding of the emission after a careful inspection of the individual spectra from both the FCRAO and IRAM $30 \mathrm{~m}$ telescopes.

The above parametrization fixes the physical structure of each core and leaves the abundance law of each molecular species as the only free parameter left to fit the observed radial profiles of intensity. Following previous work, we explored two types of abundance laws. As a first choice, we asumed that the molecular abundance is uniform across the core, and we used the Monte Carlo model to predict the intensity radial profile. Sometimes, this uniform abundance law predicted an emission profile that is much steeper than observed, and in these cases we modified the abundance law to include a central depletion hole of radius $r_{\mathrm{d}}$ within which the abundance is negligible.

As Fig. 5 shows, the $\mathrm{N}_{2} \mathrm{H}^{+}$radial profile of all the cores can be fitted using a uniform abundance model. Each core, however, requires a different abundance value. Cores $\mathrm{A} 2$ and $\mathrm{C}$ present the lowest $\mathrm{N}_{2} \mathrm{H}^{+}$abundances, with best-fit values that are four and two times lower than the value in core $\mathrm{B}$, the most $\mathrm{N}_{2} \mathrm{H}^{+}$-rich core in the sample (see Table 4 for absolute abundance values). As mentioned above, the $\mathrm{N}_{2} \mathrm{H}^{+}$abundance in a core is expected to increase with time, so we can interpret the observed differences in $\mathrm{N}_{2} \mathrm{H}^{+}$abundance as differences in the state of the chemical evolution of the cores. To better illustrate this evolution, we have re-ordered the cores in Fig. 5 so that the $\mathrm{N}_{2} \mathrm{H}^{+}$abundance decreases towards the right. If our understanding of the $\mathrm{N}_{2} \mathrm{H}^{+}$ chemistry is correct, the sequence in the figure must represent a sequence of cores of decreasing age, in which core $\mathrm{B}$ is the oldest and cores A2 and C are the youngest. (Core D has been 
placed in the sequence by considering its $\mathrm{N}_{2} \mathrm{H}^{+}(1-0)$ intensity towards the center, as no abundance estimate was possible without a mm-continuum map.)

The analysis of the SO abundance provides further support to the $\mathrm{N}_{2} \mathrm{H}^{+}$-derived age sequence. As mentioned above, $\mathrm{SO}$ is expected to behave opposite to $\mathrm{N}_{2} \mathrm{H}^{+}$, and therefore show the largest abundances in the chemically youngest cores. Indeed, Fig. 5 and Table 4 show that the young cores $\mathrm{A} 2$ and $\mathrm{C}$ have large and uniform SO abundances, while the more evolved B and $\mathrm{A} 1$ cores present $\mathrm{SO}$ depletion holes towards their center.

Finally, all cores require a depletion hole in their $\mathrm{C}^{18} \mathrm{O}$ abundance law to fit the observed emission profile. The youngest A 2 core requires the smallest hole, while the oldest B core requires the largest one (Table 4). Still, the correlation between evolutionary stage and $\mathrm{C}^{18} \mathrm{O}$ depletion hole is weak, probably because determining the correct $\mathrm{C}^{18} \mathrm{O}$ abundance law depends on modeling the poorly constrained envelope that surrounds each core. In any case, the results of the $\mathrm{C}^{18} \mathrm{O}$ analysis are consistent with the interpretation that the L1517 cores are at different stages of evolution.

Before finishing our analysis, it is worth mentioning that in the evolutionary sequence of Fig. 5, core D, for which no modeling could be carried out, appears as a relatively evolved core based both on its strong $\mathrm{N}_{2} \mathrm{H}^{+}$emission and on the flattened radial profiles of $\mathrm{SO}$ and $\mathrm{C}^{18} \mathrm{O}$. This conclusion, however, disagrees with the recent analysis by Hirota et al. (2009), who suggests that L1517D is an unusually young core from a chemical point of view. Resolving this contradiction requires making mm-continuum observations of this object to determine its true density structure and to allow a Monte Carlo modeling of its abundance profiles like that of the other cores.

Although the cores in L1517 differ significantly in their chemical composition and therefore evolutionary state, they present very similar kinematic properties. Table 3 shows that the $\mathrm{N}_{2} \mathrm{H}^{+}$linwewidths are all close to $0.2 \mathrm{~km} \mathrm{~s}^{-1}$, which is typical of the low-mass cores in Taurus and indicative of subsonic nonthermal motions (Myers 1983; Caselli et al. 2002). In addition, the internal velocity gradients of the cores are on the order of $1 \mathrm{~km} \mathrm{~s}^{-1} \mathrm{pc}^{-1}$, which is also typical of low-mass cores (Goodman et al. 1993; Caselli et al. 2002), and again suggests that the L1517 cores are representative of the population of lowmass cores in Taurus as a whole. This combination of different chemical composition and similar internal velocity structure suggests that the kinematic properties of the cores change little during their contraction from the more diffuse gas in the cloud. To further elucidate this issue, in the next sections we analyze the kinematics of the more extended gas in the cloud, and we compare it with that of the cores.

\section{Gaussian decomposition of the spectra}

In Sect. 3.1 we saw that each filament in the L1517 cloud is characterized by a well-defined range of LSR velocities (Table 1). The filaments, however, are not monolithic entities and seem to have an internal velocity structure characterized by low-level velocity gradients. As these gradients have potential information on the gas motions responsible for forming the embedded dense cores, we analyzed them in more detail by inspecting the individual $\mathrm{C}^{18} \mathrm{O}(1-0)$ spectra. From this inspection, we find that $92 \%$ of the spectra with peak intensity $S / N \geq 3$ present a single velocity component, while the rest of the spectra present evidence of two partly-overlapping velocity components. This preponderance of profiles with a well-defined number of components suggests that

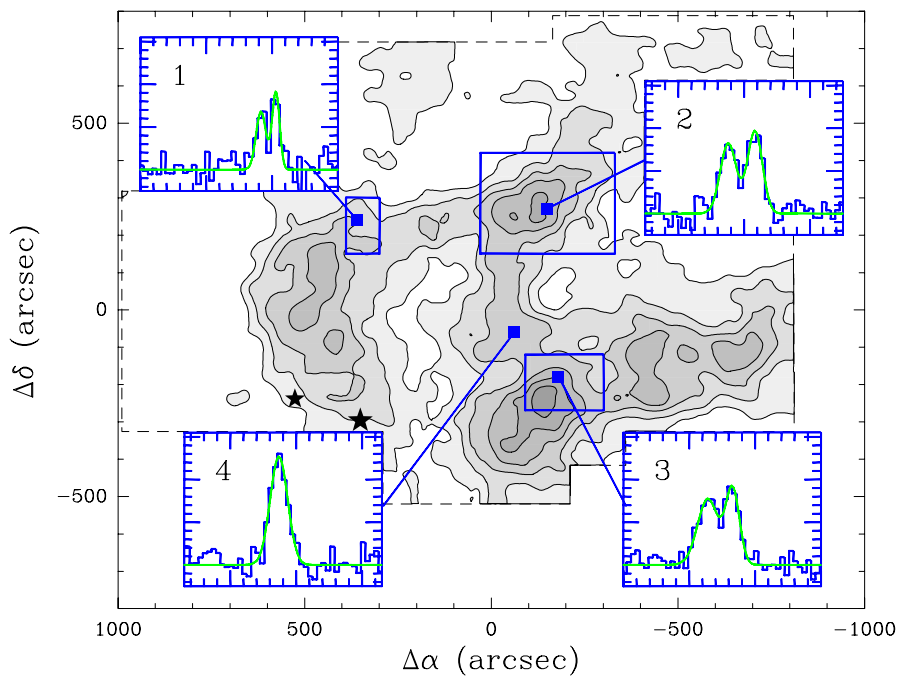

Fig. 6. Graphical summary of the Gaussian fits to the $\mathrm{C}^{18} \mathrm{O}(1-0)$ emission in L1517. The gray scale shows the $C^{18} \mathrm{O}(1-0)$ integrated intensity map of Fig. 1, and the superposed blue boxes enclose the regions where two Gaussians were fitted to the spectra. Insets with numbers 1,2 , and 3 illustrate double-Gaussian fits, while inset number 4 shows an example of the dominant single Gaussian fitted to most of the cloud spectra.

the velocity field at each cloud position can be characterized by fitting Gaussians to the spectra.

To fit the more than $2000 \mathrm{C}^{18} \mathrm{O}(1-0)$ spectra observed towards L1517 we have used a semi-automatic procedure in the CLASS software and chosen to fit one or two components depending on the complexity of the line profile. The result from this fitting is illustrated in Fig. 6, which also shows examples of the profiles. As can be seen, most of the cloud spectra have been fitted with single Gaussians (as shown in box number 4), and the fits with two Gaussians are restricted to three well-defined regions identified with solid boxes. The largest of these three regions (box number 2) is centered near $(\Delta \alpha, \Delta \delta)=$ $\left(-200^{\prime \prime},+250^{\prime \prime}\right)$ and seems to correspond to the superposition of filaments 3 and 4. This region appears in optical images as a relative enhancement in the obscuration, and Lee \& Myers (1999) even classified it as an additional core (L1517B-2), but our observations suggest that it is more likely a superposition effect. Another region with double spectra (box number 1) also seems to result from the superposition of two different components, this time filament 3 and an unlabeled and more diffuse component parallel to filament 4 that can be seen in the reddest map of Fig. 2. Finally, the third region with double spectra occurs near $(\Delta \alpha, \Delta \delta)=\left(-200^{\prime \prime},-200^{\prime \prime}\right)$ and has a less clear origin. Its location would suggest an origin in the superposition of filaments 1 and 2, but the velocity of the blue component does not match the velocity of any of the filaments. Observations with higher $S / N$ towards this region are needed to determine the origin of this anomalous component.

The Gaussian fitting procedure has also been applied to the $\mathrm{SO}$ and $\mathrm{N}_{2} \mathrm{H}^{+}$spectra. For $\mathrm{SO}$, which is almost as extended spatially as $\mathrm{C}^{18} \mathrm{O}$ (see Fig. 4), we again find that we need to use two Gaussian components inside the regions discussed before, while single-peaked spectra are the norm in the rest of the cloud. For the $\mathrm{N}_{2} \mathrm{H}^{+}(1-0)$ spectra, we fitted all hyperfine components simultaneously using the HFS method in CLASS and derived both the line centroid and the optical-depth corrected linewidth in this way. As shown in Fig. 4, the $\mathrm{N}_{2} \mathrm{H}^{+}$emission is much more compact than the $\mathrm{C}^{18} \mathrm{O}$ and $\mathrm{SO}$ emission, and our inspection of 


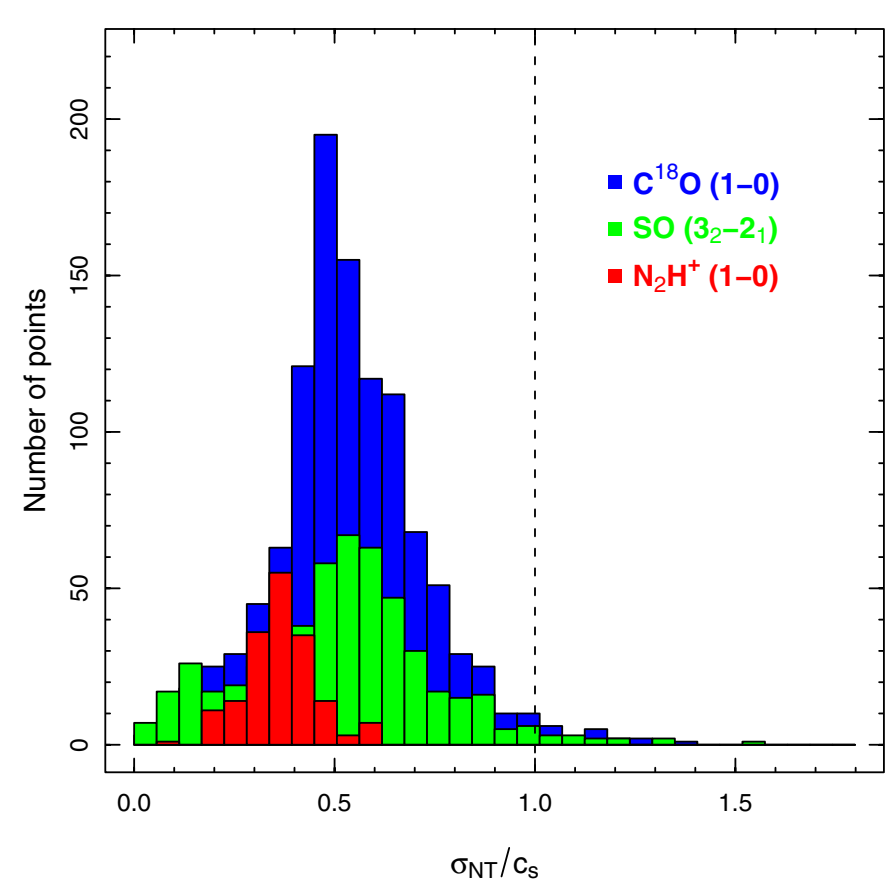

Fig. 7. Histogram of the nonthermal velocity dispersion for $\mathrm{C}^{18} \mathrm{O}$ (blue), $\mathrm{SO}$ (green), and $\mathrm{N}_{2} \mathrm{H}^{+}$(red) illustrating the predominance of subsonic values.

the individual spectra found that all positions could be fitted with a single velocity component.

The linewidths and velocity centroids derived with the Gaussian fits just described constitute the two input parameters for our analysis of the gas kinematics in L1517. In the following two sections, we analyze the behavior of each quantity separately.

\section{Linewidth analysis}

\subsection{Linewidth statistics: prevalence of subsonic motions}

The linewidth of a spectrum combines contributions from both thermal and nonthermal gas motions. In this section, we are interested in the nonthermal motions of the gas, which are potentially associated to gas turbulence or core formation motions. To isolate these motions, we have subtracted the thermal component from the measured linewidth following the standard practice of assuming that the two contributions are independent of each other so they add in quadrature (e.g., Myers 1983). In this way, we estimate the nonthermal velocity dispersion for each species as

$\sigma_{\mathrm{NT}}=\sqrt{\frac{\Delta V^{2}}{8 \ln 2}-\frac{k T}{m}}$,

where $\Delta V$ is the measured $F W H M$ linewidth, $k$ the Boltzmann's constant, $T$ the gas kinetic temperature, and $m$ the mass of the molecule under consideration. This velocity dispersion $\sigma_{\mathrm{NT}}$ can be directly compared to the (isothermal) sound speed of the gas, $c_{\mathrm{s}}$, which has a value of $0.19 \mathrm{~km} \mathrm{~s}^{-1}$ for ISM gas at $10 \mathrm{~K}$.

Figure 7 shows the histograms of the velocity dispersion for the three molecular species in our survey (using all spectra with peak intensity $S / N \geq 3$ ). As can be seen, the overwhelming majority of the spectra present a subsonic velocity dispersion and lie to the left of the $\sigma_{\mathrm{NT}} / c_{\mathrm{s}}=1$ dashed line. The few positions that exceed the sonic limit form a low-level tail in the distribution that contains $2 \%$ of the spectra in $\mathrm{C}^{18} \mathrm{O}$ and $3 \%$ of the spectra in SO. No $\mathrm{N}_{2} \mathrm{H}^{+}$spectrum has a supersonic linewidth.

In addition to a very small fraction of supersonic points, the main feature of the histograms in Fig. 7 is the presence of a well-defined central peak. This peak indicates that the gas in the cloud is not only subsonic, but it also has a favored number of nonthermal motions that approximately equal half the sound speed. More specifically, the histograms in the figure have the following mean and rms: $\sigma_{\mathrm{NT}} / c_{\mathrm{s}}=0.54 \pm 0.19$ for $\mathrm{C}^{18} \mathrm{O}$, $\sigma_{\mathrm{NT}} / c_{\mathrm{S}}=0.51 \pm 0.23$ for $\mathrm{SO}$, and $\sigma_{\mathrm{NT}} / c_{\mathrm{S}}=0.36 \pm 0.09$ for $\mathrm{N}_{2} \mathrm{H}^{+}$.

The values for the $\mathrm{C}^{18} \mathrm{O}$ and $\mathrm{SO}$ nonthermal linewidth seem consistent with each other. The mean $\sigma_{\mathrm{NT}} / c_{\mathrm{S}}$ of $\mathrm{N}_{2} \mathrm{H}^{+}$, on the other hand, is significantly smaller than that of $\mathrm{C}^{18} \mathrm{O}$ and SO. Optical depth effects could potentially play a role in this difference, since the hyperfine fit to the $\mathrm{N}_{2} \mathrm{H}^{+}(1-0)$ spectrum automatically corrects for optical depth broadening, while no correction is applied to the $\mathrm{C}^{18} \mathrm{O}$ and $\mathrm{SO}$ data when fitting single Gaussians. To test whether optical depth broadening can explain the higher $\mathrm{C}^{18} \mathrm{O}$ mean value, we have applied a simple correction to the $\mathrm{C}^{18} \mathrm{O}$ data by assuming an excitation temperature of $10 \mathrm{~K}$ and estimating the optical depth from the peak intensity of the observed line profile. This correction is relatively small, as the optical depth never reaches unity, so even after its application, the distribution of $\sigma_{\mathrm{NT}}$ still has a significantly higher mean value than for $\mathrm{N}_{2} \mathrm{H}^{+}$, and both a Kolmogorov-Smirnov and a Wilcoxon-Mann-Whitney test confirm that the two distributions must be different.

The lower $\sigma_{\mathrm{NT}}$ value of the $\mathrm{N}_{2} \mathrm{H}^{+}$spectra could indicate that the dense core gas has an intrinsically lower velocity dispersion than the less-dense ambient material and that core formation has been accompanied by certain amount of dissipation of nonthermal motions. If this is the case, we can quantify the loss of nonthermal motions from the difference between the FWHM linewidths of $\mathrm{C}^{18} \mathrm{O}$ and $\mathrm{N}_{2} \mathrm{H}^{+}$, which is $0.07 \pm 0.04 \mathrm{~km} \mathrm{~s}^{-1}$.

Even if small, the above estimate of the loss of nonthermal motions during core formation is likely a significant overestimate. The nonthermal linewidth is not a local parameter, but the accumulated effect of gas motions along the line of sight. For this reason, some consideration should be given to line-of-sight effects when comparing tracers as different as $\mathrm{C}^{18} \mathrm{O}$ and $\mathrm{N}_{2} \mathrm{H}^{+}$. As shown in Sect. 4.1, the $\mathrm{N}_{2} \mathrm{H}^{+}$emission is significantly more concentrated than the emission from $\mathrm{C}^{18} \mathrm{O}$ and $\mathrm{SO}$ because of its special chemistry and excitation requirements, so it samples a shorter line of sight path than $\mathrm{C}^{18} \mathrm{O}$ and SO. In addition, we see in the next section that part of the kinematics of the $\mathrm{C}^{18} \mathrm{O} / \mathrm{SO}$ emitting gas arises from large-scale motions in the filaments. As these motions likely accumulate along the line of sight, they are expected to contribute more to the $\mathrm{C}^{18} \mathrm{O}$ and $\mathrm{SO}$ linewidth than to $\mathrm{N}_{2} \mathrm{H}^{+}$.

To estimate the importance of this effect, we compare the sizes of the typical emitting regions for $\mathrm{N}_{2} \mathrm{H}^{+}$and $\mathrm{C}^{18} \mathrm{O}$. Tables 2 and 4 show that the typical diameter of an L1517 core traced in $\mathrm{N}_{2} \mathrm{H}^{+}$is about $2 \times 55^{\prime \prime}$, while the same parameter for a filament traced in $\mathrm{C}^{18} \mathrm{O}$ is about $2 \times 95^{\prime \prime}$. In addition, Table 5 shows that the typical velocity gradient in the filament gas is about $1.2 \mathrm{~km} \mathrm{~s}^{-1} \mathrm{pc}^{-1}$. Thus, if we assume a gradient of this size and multiply it by the difference in line of sight length between the two molecules, we predict a velocity difference between the tracers due to large-scale motions of about $0.06 \mathrm{~km} \mathrm{~s}^{-1}$. This difference is so close to the observed linewidth difference that it seems very likely that a significant part of the nonthermal linewidth difference between $\mathrm{N}_{2} \mathrm{H}^{+}$and $\mathrm{C}^{18} \mathrm{O}$ (and $\mathrm{SO}$ ) arises from their different sampling of the cloud large-scale motions. Whether this 
Table 5. Kinematic properties of the filaments ${ }^{1}$.

\begin{tabular}{lccc}
\hline \hline Filament & $\left\langle\sigma_{\mathrm{NT}}\right\rangle / c_{\mathrm{s}}$ & $\begin{array}{c}\left\langle V_{\mathrm{LSR}}\right\rangle \\
\left(\mathrm{km} \mathrm{s}^{-1}\right)\end{array}$ & $\begin{array}{c}\left\langle\left|\nabla V_{\mathrm{LSR}}\right|\right\rangle \\
\left(\mathrm{km} \mathrm{s}^{-1} \mathrm{c}^{-1}\right)\end{array}$ \\
\hline 1 & $0.57 \pm 0.15$ & $5.52 \pm 0.07$ & $1.0 \pm 0.5$ \\
2 & $0.63 \pm 0.17$ & $5.79 \pm 0.07$ & $1.4 \pm 0.7$ \\
3 & $0.53 \pm 0.16$ & $5.89 \pm 0.05$ & $1.3 \pm 1.0$ \\
4 & $0.41 \pm 0.16$ & $6.14 \pm 0.04$ & $0.9 \pm 0.5$ \\
\hline
\end{tabular}

Notes. ${ }^{(1)}$ From $\mathrm{C}^{18} \mathrm{O}(1-0)$ emission.

effect can explain the full linewidth difference cannot be said, but it does show that the difference in local nonthermal motions (and thus dissipation during core formation) must be necessarily less than estimated before.

A simple consequence of the subsonic nature of the nonthermal gas motions in L1517 is the small role that they can play in supporting the cloud against gravity. The ratio between the nonthermal and thermal contributions to the gas pressure is given by $P_{\mathrm{NT}} / P_{\mathrm{T}}=\left(\sigma_{\mathrm{NT}} / c_{\mathrm{s}}\right)^{2}$, so even if we use the $\mathrm{C}^{18} \mathrm{O}$ linewidth uncorrected for optical depth, we find that the nonthermal motions contribute to the gas pressure with only $\sim 1 / 3$ of the thermal value. This contribution is likely to represent an upper limit, because the above pressure ratio assumes again that all nonthermal motions arise from a local "microscopic" turbulent motions that increases cloud support (Chandrasekhar 1951) and ignores the contribution from large-scale flows.

\subsection{Spatial distribution and consequences for turbulent models of core formation}

To further characterize the nonthermal motions, we now study their spatial distribution. Figure 8 presents a plot of $\sigma_{\mathrm{NT}} / c_{\mathrm{S}}$ for $\mathrm{C}^{18} \mathrm{O}$ and $\mathrm{N}_{2} \mathrm{H}^{+}$as a function of position along each of the filaments identified in Sect. 3.1. In agreement with the histogram analysis, all four filaments are dominated by subsonic gas over their entire length, and only a few positions exceed the sonic limit in $\mathrm{C}^{18} \mathrm{O}$. These few supersonic positions (some of them coincident with filament overlaps) appear almost randomly scattered over the filaments length, and show no correlation with either the position of the cores or the beginning or end of the filaments.

In addition to a lack of supersonic points, the plots of nonthermal linewidth in Fig. 8 present very little spatial structure. Filaments 1, 3, and 4 have almost flat distributions of $\mathrm{C}^{18} \mathrm{O}$ $\sigma_{\mathrm{NT}} / c$ and well-defined mean values close to 0.5 (Table 5). Filament number 2 also has a mean $\sigma_{\mathrm{NT}} / c$ close to 0.5 , but presents changes in the linewidth along its length. These changes are of unclear origin, and seem to result from the presence of two localized regions of enhanced (but mostly subsonic) linewidth. One of these regions corresponds to the vicinity of core B, and the other occurs in the vicinity of core A1. Concerning the $\mathrm{N}_{2} \mathrm{H}^{+}$linewidths, Fig. 8 again shows their tendency to be smaller than those of $\mathrm{C}^{18} \mathrm{O}$. This trend is present in all the cores, although it is somewhat reduced in core D of filament 3. Apart from this already discussed effect, Fig. 8 shows that the $\mathrm{N}_{2} \mathrm{H}^{+}$linewidths remain approximately constant inside each of the cores.

The flat distribution of the velocity dispersion in the filaments, and in particular in the vicinity of the dense cores, poses a significant constraint to models of core formation by supersonic turbulence. In these models, the cores are shock-compressed structures formed at the stagnation point between two convergent flows, and as a result, they are expected to be surrounded

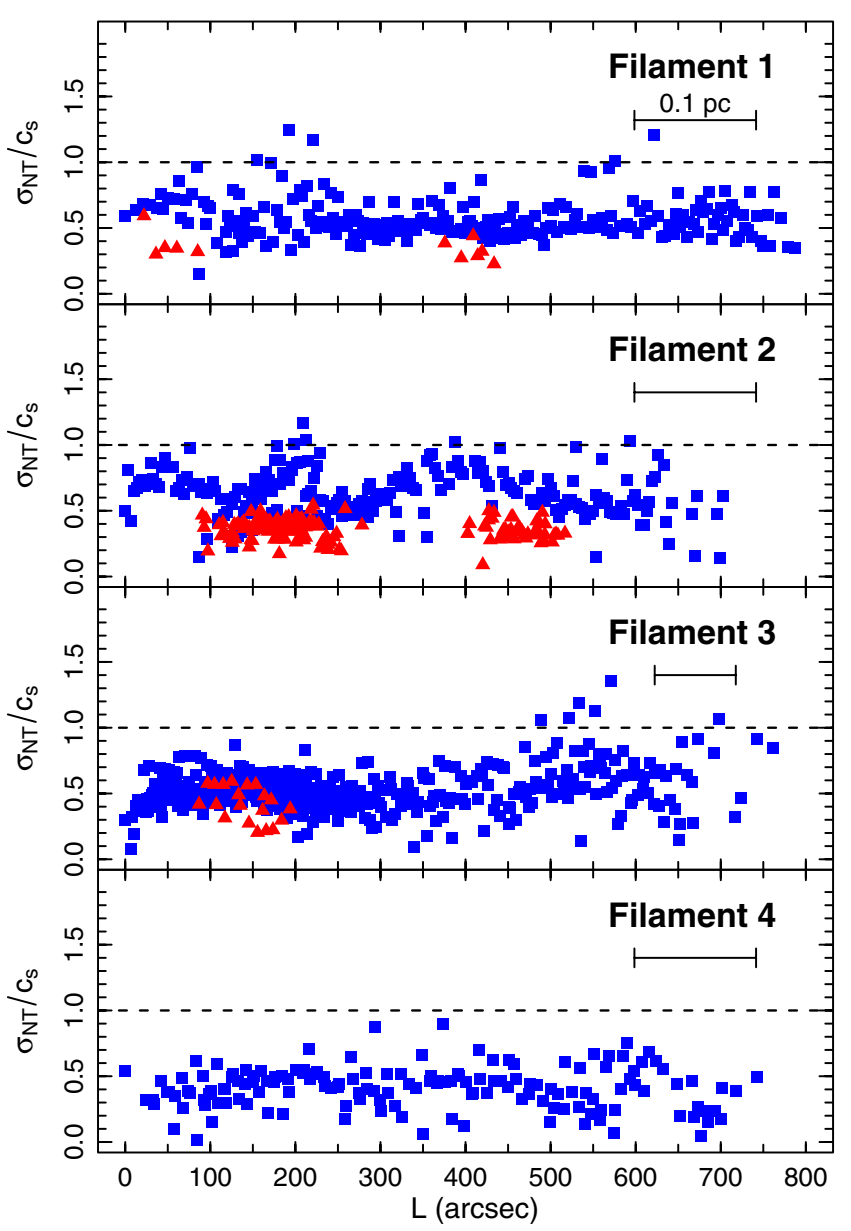

Fig. 8. Distribution of nonthermal velocity dispersion as a function of position along each of the $\mathrm{L} 1517$ filaments. Blue squares represent $\mathrm{C}^{18} \mathrm{O}(1-0)$ and red triangles and $\mathrm{N}_{2} \mathrm{H}^{+}(1-0)$ values. The spatial scale for filament 3 has been shrunk by a factor of 1.5 in order to fit it into the reduced box size used for the other filaments. The mean formal error in the $\sigma_{\mathrm{NT}} / c_{\mathrm{s}}$ determination is 0.1 for both $\mathrm{N}_{2} \mathrm{H}^{+}$and $\mathrm{C}^{18} \mathrm{O}$, which is approximately the marker size.

(even confined) by turbulent layers of cloud gas (e.g., Padoan et al. 2001; Ballesteros-Paredes et al. 2003; Klessen et al. 2005). In $\mathrm{L} 1517$, the observed $\mathrm{C}^{18} \mathrm{O}$ emission surrounds the location of the dense cores and arises from gas that is about one order of magnitude less dense than the core centers (Sect. 4.1), so we can naturally expect that the $\mathrm{C}^{18} \mathrm{O}$ emission arises from the gas layers that physically surround the dense cores. $\left(\mathrm{C}^{18} \mathrm{O}\right.$ does not sample gas inside the cores due to freeze out, see Sect. 4.) If the cores have formed by the convergence of gas flows, part of the $\mathrm{C}^{18} \mathrm{O}$ emission must originate in gas in the flows and must retain a signature from the core-forming convergent motions. To test whether such a signature is present in our data, we calculated for each core the average $\mathrm{C}^{18} \mathrm{O}$ velocity dispersion inside rings around the core center having radii equal to $1,2,3$, and 4 core radii (core radius is $r_{1 / 2}$ in Table 4 ). This procedure is illustrated in the upper panel of Fig. 9, and has been stopped at four core radii to ensure that we sample equally gas that surrounds the cores in all directions, and not only in the direction of the large-scale filaments (which dominates the emission at larger radii). The results from this average for each of the five cores in L1517 are indicated in the bottom panel of Fig. 9. Not surprisingly given the general trend seen in the filaments, all cores 


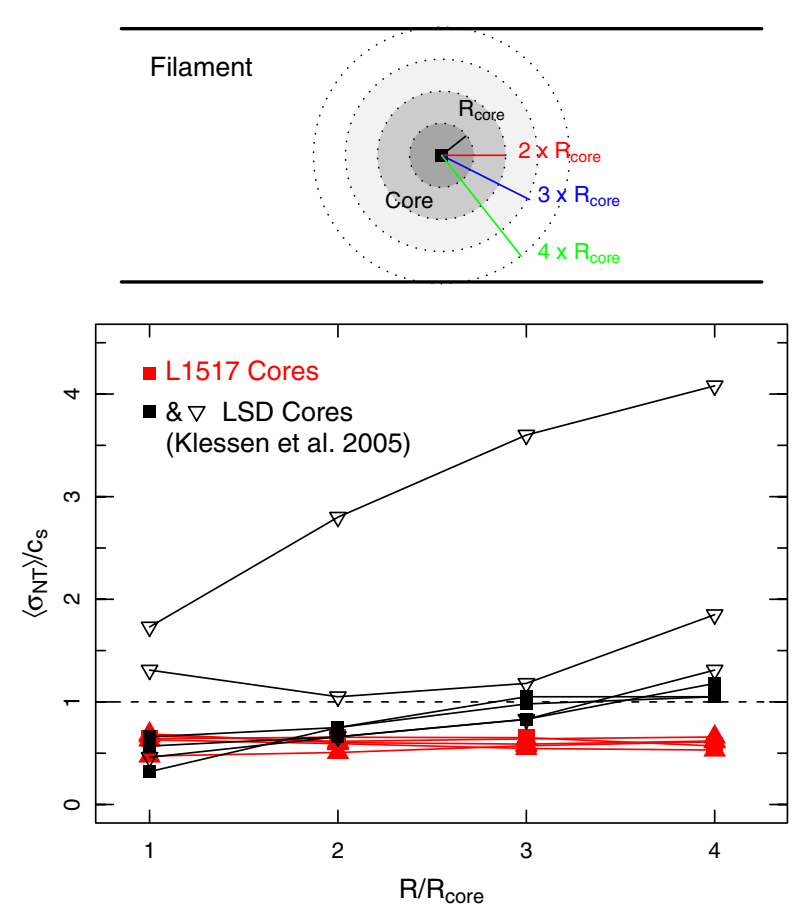

Fig. 9. Comparison between the $\mathrm{C}^{18} \mathrm{O}$ nonthermal velocity dispersion around each L1517 core and the predictions from a model of turbulent core formation. Top: schematic view illustrating how the velocity dispersion has been averaged inside four concentric shells around each core. Bottom: comparison between the observed $\mathrm{C}^{18} \mathrm{O}$ data (red solid triangles) and the predictions from the LSD model by Klessen et al. (2005). The black solid squares represent the three different projections of a fully subsonic core, while the open inverted triangles represent the three projections of a core that has transonic motions in one of them.

present a distribution of velocity dispersion with radius that has no systematic change with distance from the core center.

To compare the distribution of velocity dispersions around the cores of L1517 with the predictions from turbulent models, we chose the numerical simulations of gravo-turbulent core formation by Klessen et al. (2005). These authors attempt to reproduce conditions similar to those of a low-mass star-forming region like L1517 and present a very complete view of the internal kinematics of the simulated cores. From the two families of simulations that these authors present, we selected the largescale driving (LSD) case because it produces the most quiescent cores, and is therefore more likely to fit our observations of L1517. Klessen et al. (2005) present in their Fig. 1 radial profiles of the velocity dispersion for three different views of two selected cores (likely chosen for their similarity to observations), and we used these profiles to derive nonthermal velocity components by subtracting in quadrature the contribution from thermal motions (as done with the $\mathrm{C}^{18} \mathrm{O}$ data). These data have been averaged as a function of core radius by converting the normalized column densities given by the authors into a distance from the core center by assuming that the the density of the cores follows the profile of a Bonnor-Ebert sphere (as claimed by the authors).

The predicted nonthermal velocity dispersions from the Klessen et al. (2005) model are represented in Fig. 9. As expected, these predicted dispersions show a significant increase with radius due to the presence of converging flows around the cores. The predicted increase is approximately a factor of 2 between 1 and 4 core radii, and as a result, the outermost points in the graph are expected to exceed the sound speed limit in all core models. Even in the most quiescent, subsonic core model, which is arguably not representative of the sample because subsonic cores are less than $25 \%$ of the total in the simulation, the match between model and data is only acceptable for the innermost two radii. For larger radii, model and data diverge with increasing distance from the core center, and end up differing by a factor of 2 in the outermost layers sampled by our observations. Even larger disagreement occurs for the case of transonic cores, which constitute $\sim 50 \%$ of the total cores in the simulation and are therefore more representative of the model results. As can be seen, two out of the three sets of points exceed the sound speed at all radii, and therefore fail to fit the observations at all radii in all the cores.

Although limited, the above comparison illustrates the basic disagreement between our observations of the L1517 cloud and the predictions from models of core formation by convergence of supersonic flows: the $\mathrm{C}^{18} \mathrm{O}$ spectra in L1517 sample the core outer layers far enough to trace any core-forming motions, so if these motions were supersonic, they should have left a clear signature in the $\mathrm{C}^{18} \mathrm{O}$ spectra. The absence of such a signature rules out the presence supersonic motions around the cores and sets a limit to any core-forming gas flow that is well inside the subsonic regime.

\subsection{Velocity coherent filaments}

In their study of the linewidth of dense-gas tracers in cores, Barranco \& Goodman (1998) and Goodman et al. (1998) found that the nonthermal component remains almost constant over the core interior and that this behavior represents a departure from the well-known linewidth-size relation commonly associated to turbulent motions (Larson 1981). These authors refer to this property of the core gas as velocity "coherence", and suggest that the scale of coherence, which they found to approximately coincide with the core size, may indicate that core formation is related to the process of turbulence dissipation.

Our analysis of the $\mathrm{C}^{18} \mathrm{O}$ linewidth illustrated in Figs. 8 and 9 shows that the region with constant nonthermal linewidth can be followed in significantly larger scales than the cores and that it extends over distances as long as the filaments themselves $(\sim 0.5 \mathrm{pc})$. This coherence of the velocity field in the filaments is not limited to the nonthermal linewidth, and it can be seen in the behavior of the velocity centroid, to be studied in detail in the next section. In the L1517 cloud, therefore, the filaments are velocity coherent in the sense of Barranco \& Goodman (1998) and Goodman et al. (1998), and this extends the scale size of coherence in the gas by a factor of approximately 5 .

Finding velocity coherence on scales as large as the filaments separates the scale of coherence from that of the cores, and suggests that core formation is not likely the direct result of turbulence dissipation. As seen in Sect. 6.1, some turbulence dissipation may have occurred during core formation, but by being significantly subsonic, it is unlikely to have affected the gas pressure balance and triggered core formation. Velocity coherence in cores, therefore, does not seem a defining property of the condensations, but a condition inherited from the larger spatial scale of the filaments.

Our finding of velocity coherence on scales larger than a core would seem to contradict the recent results from Pineda et al. (2010), who claim to have detected of the transition between the coherent and turbulent regimes in a core with observations of the B5 region in Perseus. We note, however, that although these authors associate the velocity coherent region with a dense core, one can see in their Fig. 3 that the velocity coherent region 


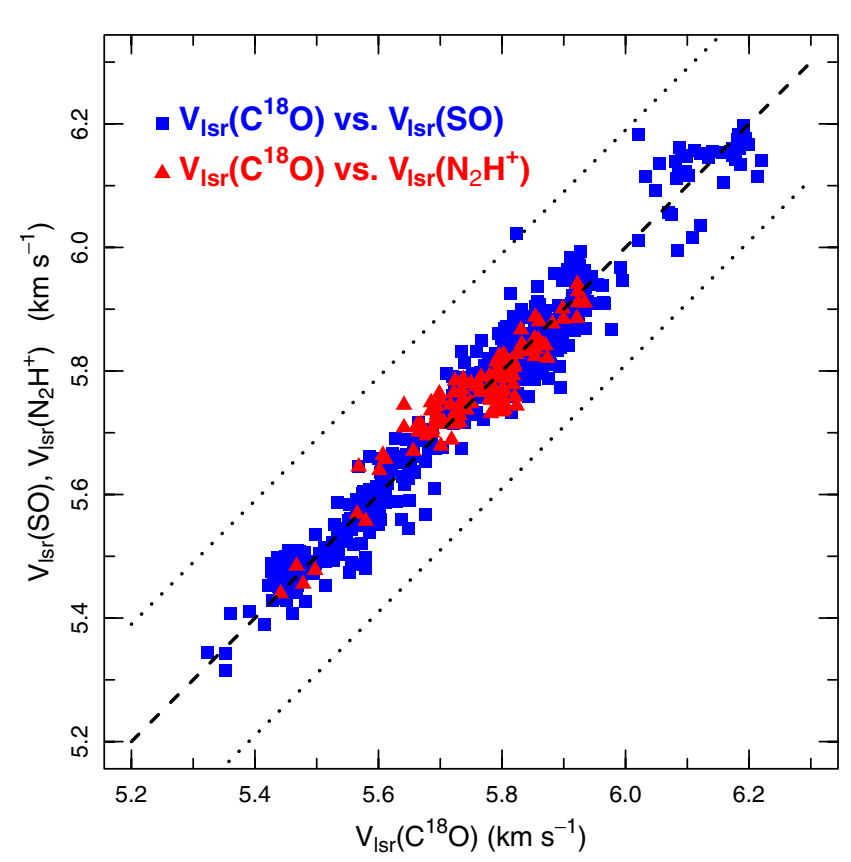

Fig. 10. Comparison between the $V_{\mathrm{LSR}}$ of $\mathrm{C}^{18} \mathrm{O}$ and $\mathrm{SO}$ (blue squares), and $\mathrm{C}^{18} \mathrm{O}$ and $\mathrm{N}_{2} \mathrm{H}^{+}$(red triangles) in L1517 illustrating the good agreement between all tracers. The central dashed line indicates the locus of equal velocities, and the surrounding dotted lines have been displaced by the sound speed $\left(0.19 \mathrm{~km} \mathrm{~s}^{-1}\right.$ for ISM gas at $\left.10 \mathrm{~K}\right)$. The mean formal error in the $V_{\mathrm{LSR}}$ determination is $0.02 \mathrm{~km} \mathrm{~s}^{-1}$ for $\mathrm{SO}$ and $0.01 \mathrm{~km} \mathrm{~s}^{-1}$ for both $\mathrm{C}^{18} \mathrm{O}$ and $\mathrm{N}_{2} \mathrm{H}^{+}$, which is comparable or smaller than the marker size.

in B5 is elongated and has a length of about $0.5 \mathrm{pc}$, similar to our L1517 filaments. The B5 region, in addition, contains multiple dense cores (Enoch et al. 2006, also our own unpublished data), and this suggests again that it is more similar to the L1517 filaments than to an isolated dense core. Clearly more observations of different core environments are needed to understand the size and relation between the different velocity coherent regions. If the L1517 cloud is a representative region of dense core formation, we can predict that filament-wide velocity coherence will be a common phenomenon.

\section{Line centroid analysis}

The second output from our Gaussian fit to the spectra is the velocity centroid. Figure 10 compares the $\mathrm{SO}$ and $\mathrm{N}_{2} \mathrm{H}^{+}$centroids with those of $\mathrm{C}^{18} \mathrm{O}$ towards all positions where the fits were considered to be significant $(S / N \geq 3)$. As can be seen, the velocities of the different tracers agree with each other independently of any variations in the bulk velocity of the cloud. The average difference in velocity between $\mathrm{C}^{18} \mathrm{O}$ and either SO or $\mathrm{N}_{2} \mathrm{H}^{+}$is $0.03 \pm 0.03 \mathrm{~km} \mathrm{~s}^{-1}$, which means that the velocities of the different tracers differ on average by less than one fifth of the sound speed. This good match between tracers rules out any significant motions between the different density regimes of the gas and, in particular, it rules out any systematic drift between the dense cores (traced by $\mathrm{N}_{2} \mathrm{H}^{+}$) and the surrounding gas (traced by $\mathrm{C}^{18} \mathrm{O}$ ). Such a quiescent state of the gas is not peculiar to L1517, and Walsh et al. (2004) and Kirk et al. (2007a) have found a similar lack of velocity shifts between $\mathrm{N}_{2} \mathrm{H}^{+}$and $\mathrm{C}^{18} \mathrm{O}$ in a number of low-mass star-forming regions.

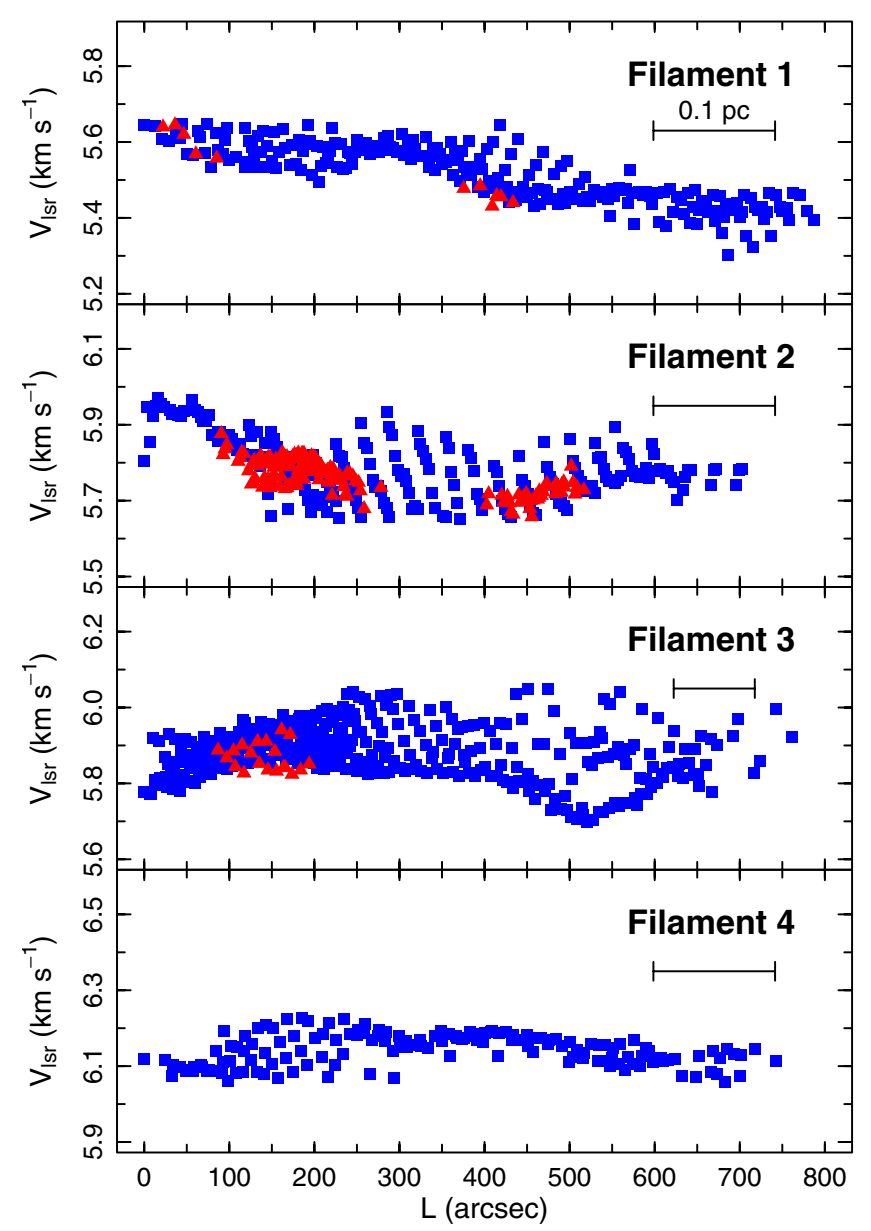

Fig. 11. Velocity centroid as a function of position along each of the L1517 filaments. Blue squares represent $\mathrm{C}^{18} \mathrm{O}(1-0)$ and red triangles $\mathrm{N}_{2} \mathrm{H}^{+}(1-0)$ values. The spatial scale for filament 3 has been shrunk by a factor of 1.5 in order to fit it into the reduced box size used for the other filaments. The mean formal error in the velocity centroid determination is $0.02 \mathrm{~km} \mathrm{~s}^{-1}$ for $\mathrm{C}^{18} \mathrm{O}$ and $0.01 \mathrm{~km} \mathrm{~s}^{-1}$ for $\mathrm{N}_{2} \mathrm{H}^{+}$, which is always smaller than the marker size.

\subsection{Continuity of the velocity field and large-scale oscillations}

The lack of velocity shifts between tracers does not mean that the gas in L1517 is static. Figure 10 shows how the LSR velocity of the cloud material spans almost $1 \mathrm{~km} \mathrm{~s}^{-1}$, and this velocity spread arises from a combination of the different velocities of each of the filaments and the presence of internal velocity gradients inside the filaments. To more fully study these gradients, we present plots of the velocity centroids of $\mathrm{C}^{18} \mathrm{O}$ and $\mathrm{N}_{2} \mathrm{H}^{+}$as a function of position along each filament in Fig. 11.

As can be seen in Fig. 11, the velocity field of each filament presents a remarkably low level of spatial change. The dispersion of the $\mathrm{C}^{18} \mathrm{O}$ velocity centroids does not exceed $0.07 \mathrm{~km} \mathrm{~s}^{-1}$ in any filament, even when considering a few regions of enhanced dispersion that seem to coincide with locations of multiplepeaked spectra and to overlap between different filaments. In addition, the end-to-end velocity change in each filament is about $0.2 \mathrm{~km} \mathrm{~s}^{-1}$ or less. This quiescent state of the gas on scales as large as $0.5 \mathrm{pc}$ once more justifies the interpretation of the filaments as velocity-coherent structures. 
Although the changes in velocity along the filaments are small, the low dispersion of the centroid data allows the existence of large-scale velocity patterns to be discerned in some of the filaments. Figure 11 shows how the $\mathrm{C}^{18} \mathrm{O}$ centroids oscillate quasi-periodically, especially in filaments 1 and 2, over the length of the filaments with typical wavelengths of approximately $0.1-0.2 \mathrm{pc}$. As also shown in the figure, the more spatially localized $\mathrm{N}_{2} \mathrm{H}^{+}$centroids follow the $\mathrm{C}^{18} \mathrm{O}$ oscillatory pattern at those positions where both tracers can be observed (i.e., towards the dense cores), indicating that both species trace the same general velocity pattern. This good match between the $\mathrm{C}^{18} \mathrm{O}$ and $\mathrm{N}_{2} \mathrm{H}^{+}$data is remarkable because the two species do not coexist spatially due to their anticorrelated chemistries (Sect. 4), and they therefore trace significantly different regimes of gas density. Thus, the continuity of the $\mathrm{C}^{18} \mathrm{O}$ and $\mathrm{N}_{2} \mathrm{H}^{+}$velocity gradients must reflect a continuity between the velocity field of the filament gas and the internal velocity gradients of the dense cores embedded in it. This implies that the internal velocity gradients in the cores are not intrinsic core properties (like isolated rotation), but result from the large-scale motions of surrounding filament. Core velocity gradients should therefore be interpreted in terms of the velocity gradients of the lower density gas.

The observed continuity between the velocity field of the filaments and the cores implies that core formation in L1517 has not decoupled the dense kinematically gas from its surrounding environment. This behavior again seems to contradict the expectation from the turbulent models of core formation, because in these models, the cores are formed by the direct shock of streams of lower density gas, so a discontinuity in the velocity field is expected. The observations of L1517 are better understood if the filaments are coherent in velocity as a whole, and the cores have formed from the contraction of this velocity-coherent gas. The transition from cloud to core conditions must have therefore involved little or no dissipation of kinetic energy, and in particular the absence of supersonic shocks. The data therefore indicates that core formation in L1517 has been an almost quasi-static process.

\subsection{A simple model of the velocity oscillations}

The velocity oscillations of filaments 1 and 2, dominate the large-scale kinematics of the gas in these objects, and suggest some type of ordered pattern in their three-dimensional velocity field. Our limited observations cannot fully constrain the 3D properties of this velocity field, but the large-scale nature of the motions and the lack of systematic velocity gradients perpendicular to the filament axis suggest that the underlying velocity field must have an important component directed along the axis of the filaments. If this is correct, it is intriguing to investigate whether the velocity oscillations of filaments 1 and 2 are related to the presence of embedded cores, and in particular, whether they could represent part of the motions responsible for core formation.

Core formation in a filamentary gas cloud is often investigated using the idealized geometry of an infinitely-long, axiallysymmetric gas cylinder. The stability of such a configuration has been studied in detail using both semi-analytical techniques (Stodólkiewicz 1963; Ostriker 1964; Larson 1985; Nagasawa 1987; Inutsuka \& Miyama 1992; Nakamura et al. 1993; Gehman et al. 1996; Fiege \& Pudritz 2000a) and numerical simulations (Bastien 1983; Bastien et al. 1991; Nelson \& Papaloizou 1993; Nakamura et al. 1995; Fiege \& Pudritz 2000b). From this body of work we know that, depending on the initial equilibrium state of the gas, the amount of support provided by pressure and magnetic fields, and the geometry of both the magnetic field and the perturbation applied to the system, the gas cylinder can follow a number of evolutionary paths, including expansion, collapse to a spindle, or fragmentation into multiple clumps. Among these outcomes, cloud fragmentation is the most interesting here because of its possible relation to core formation and the velocity oscillations in filaments 1 and 2 .

In the simplest case of an isothermal cylinder in equilibrium, fragmentation occurs through a Jeans-type of instability in which a density perturbation with a large-enough wavelength accumulates enough mass in each over-dense region to make it gravitationally unstable (e.g., Stodólkiewicz 1963; Larson 1985). The most unstable perturbation is an axisymmetric mode that breaks the initially continuous filament into a chain of dense condensations equally spaced along the cylinder axis (Nakamura et al. 1993). Numerical simulations show that this process involves redistribution of the gas in the filament via motions that have a dominant velocity component parallel to the filament axis, at least during the first stages of evolution (e.g., Nakamura et al. 1993; Fiege \& Pudritz 2000b). The similarity of these motions with those inferred for filaments 1 and 2 in L1517 is the main motivation for attempting a simple kinematic model.

Modeling in detail the fragmentation of the L1517 filaments exceeds the scope of this paper, and would require information on so far unknown cloud properties like the strength and geometry of the magnetic field. For this reason, we limit ourselves to testing whether the velocity oscillations of Fig. 11 are consistent with large-scale core-forming motions, and we do so with a highly idealized infinite cylindrical model. Our first approximation is to only study motions along the central axis of the cylinder, which allows us to convert the problem to one dimension and which is motivated by the dominance of longitudinal motions in simulations. Following standard practice (e.g., Binney \& Tremaine 1987, their Sect. 5.1), we use perturbation analysis and assume that the gas starts from a state of hydrostatic equilibrium in which the density is constant along the filament axis (e.g., Ostriker 1964). To this equilibrium state we added a small longitudinal perturbation in both density and velocity, and work only to first order in the perturbation quantities (linear analysis). Under these conditions, the equation of continuity can be written as

$\frac{\partial \rho_{1}}{\partial t}+\rho_{0} \frac{\partial u_{1}}{\partial z}=0$

where $\rho$ is the density, $u$ the velocity, $z$ the spatial coordinate along the filament axis, and the 0 and 1 subscripts refer to the unperturbed solution and the first-order perturbation. If we now assume Fourier-component perturbations for both density and velocity, we can write

$\rho_{1}(z, t)=C_{1} \mathrm{e}^{\mathrm{i}(k z-\omega t)} \quad$ and $\quad u_{1}(z, t)=C_{2} \mathrm{e}^{\mathrm{i}(k z-\omega t)}$,

where $C_{1}$ and $C_{2}$ are constants due to the lack of $z$-dependence of the unperturbed solution. Substituting these expressions into the equation of continuity, we can obtain a simple relation between the two constants. The case of interest here is that of an unstable, core-forming mode (i.e., opposed to a stable sonic wave), and this implies that $\omega^{2}$ must be negative. Defining a real number $\gamma$ so $\omega=\mathrm{i} \gamma$, we can write

$C_{2}=\mathrm{i} \frac{C_{1}}{\rho_{0}} \frac{\gamma}{k}$

As $\rho_{0}, \gamma$, and $k$ are real numbers, the above equation implies that the Fourier component of the velocity perturbation must 

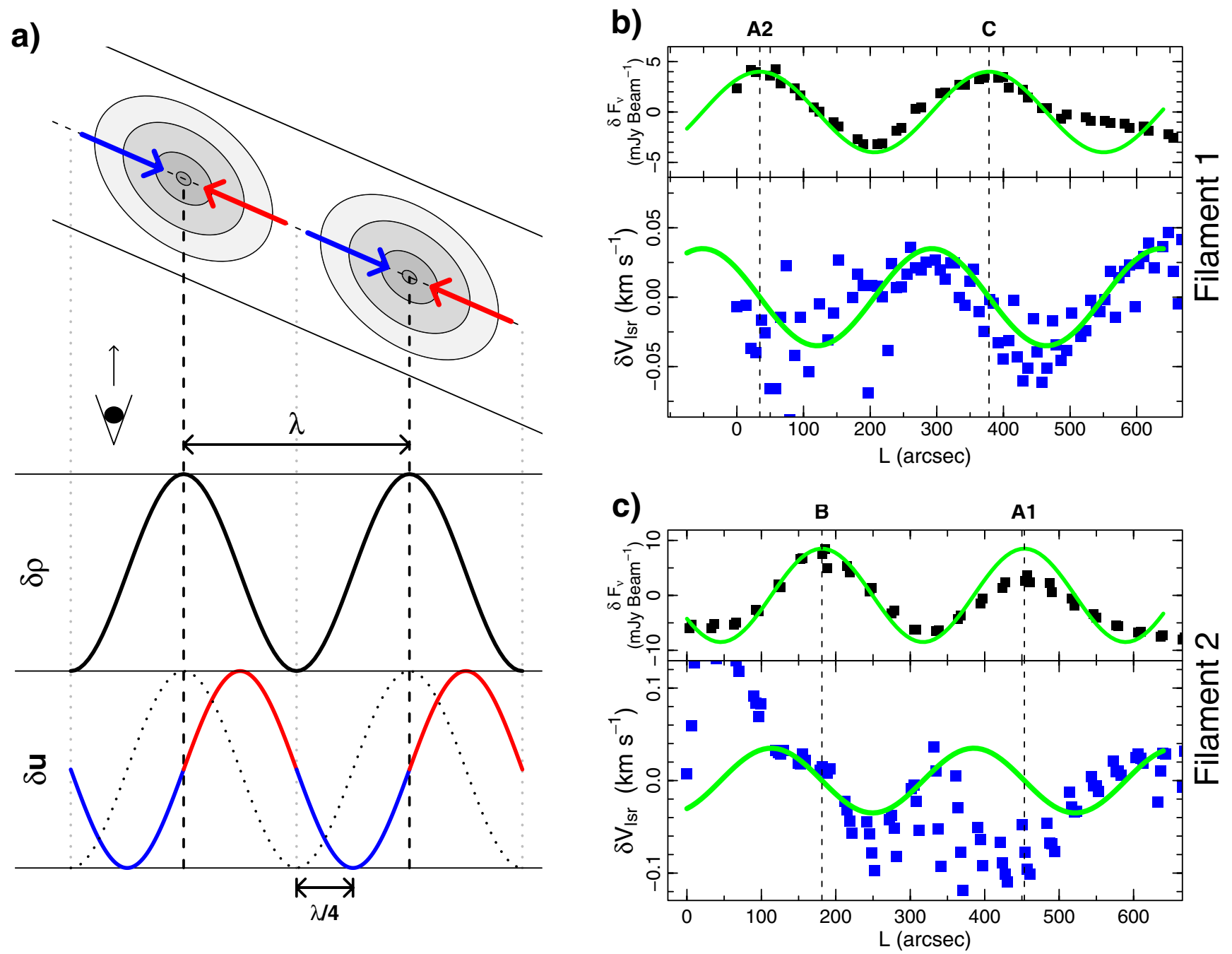

Fig. 12. Kinematic modeling of the velocity oscillations in filaments 1 and 2. a) Schematic view showing how a core-forming velocity field along the filament axis causes a $\lambda / 4$ shift between the sinusoidal perturbations of density and velocity. b), c) Comparison of the density and velocity perturbations in filaments 1 and 2 with the expectation from the simple kinematic model. For each filament, the upper panel (black squares) shows the increment of mm continuum flux over the mean (a proxy for the density perturbation), and the lower panel (blue squares) shows the variation in the $\mathrm{C}^{18} \mathrm{O}$ centroid velocity over the filament mean (after subtraction of a linear gradient). The green solid line in each upper panel is a sinusoidal fit to the density perturbation, from which a wavelength and phase are determined. The green solid line in each bottom panel is the result of shifting the density sinusoid by $\lambda / 4$ (plus scaling it arbitrarily), and represents the expected pattern for a core-forming velocity field. The mean formal error in the $\delta V_{\mathrm{LSR}}$ determination is $0.01 \mathrm{~km} \mathrm{~s}^{-1}$ for both filaments, which is on the order of the marker size.

be shifted in phase from the density component by $\pi / 2$, which is one quarter of the wavelength of the perturbation (see also Gehman et al. 1996). (In a stable (sonic) perturbation, $\omega$ is a real quantity, so the density and velocity Fourier components are in phase.)

The origin of the $\lambda / 4$ shift between the velocity and density perturbations can be easily understood with the simplified cartoon of Fig. 12a. As can be seen, for the gas motions to be core-forming, they have to converge towards the core centers, and this means that a density peak must correspond to a position of vanishing velocity. Assuming that both density and velocity perturbations are sinusoidal, this requires that there is a $\lambda / 4$ shift between the two.

The expected $\lambda / 4$ shift between the density and the velocity patterns in an unstable perturbation provides a simple criterion to test whether the velocity field in the L1517 filaments is consistent with core-forming motions. To this end, we have fitted a sinusoid to a cut of the mm-continuum emission along the filament axis. This quantity should be proportional to the filament density profile, with the caveat that the bolometer observation filters the extended emission due to chopping, and from this fit we have determined the wavelength and phase of the density perturbation in filaments 1 and 2. The top panels of Figs. 12b and c show that both filaments can be reasonably fitted with sinusoidal density profiles that have wavelengths of approximately 340 and 270 arcsec, respectively.

To now test whether the observed velocity field is consistent with the formation of these density profiles, we shift the density sinusoid by one quarter of the wavelength, as required by the perturbation analysis, and compare the result with the velocity centroid data towards the filament axis. For this step, we have one degree of freedom in the choice of the sign of the shift, as there are two possible inclinations of the filament with respect to the plane of the sky (e.g., the eastern part of filament 1 could be inclined toward us or away from us), and this adds a sign ambiguity to the gas radial velocity. When this inclination is chosen 
(together with a scaling of velocity amplitude), we derive the model predictions shown in the bottom panels of Figs. 12b and c superposed to the observed velocity centroids (to which we have subtracted global linear gradients of $0.5 \mathrm{~km} \mathrm{~s}^{-1} \mathrm{pc}^{-1}$ in filament 1 and $0.1 \mathrm{~km} \mathrm{~s}^{-1} \mathrm{pc}^{-1}$ in filament 2).

As can be seen, the velocity field of filament 1 agrees reasonably well with the prediction from the shifted sinusoid both in wavelength and phase, suggesting that the observed velocity oscillation is consistent with core-forming motions. The velocity towards core $\mathrm{C}$ does indeed coincide with a zero value in the velocity sinusoid, while the behavior of the velocity towards core A2 is not as clear due to the higher scatter and the few velocity points. Such a reasonable fit is encouraging for an interpretation of the velocity field in terms of fragmentation, as this filament is also the best behaved according to both the maps (Fig. 2) and the radial profiles (Fig. 3). It also harbors the chemically youngest cores of the cloud (Fig. 5), suggesting that it is at the earliest phases of fragmentation.

In contrast to filament 1 , filament 2 does not fit the shifted sinusoid velocity pattern as well. On the one hand, the velocity field of this filament does not follow a sinusoid pattern very closely, and on the other, a region of high scatter between cores B and A1 leaves the velocity poorly defined, especially in the vicinity of core A1. Still, as the figure shows, the position of core $\mathrm{B}$ lies in a region consistent to have zero velocity, which is the expected pattern for core-forming motions.

So far we have concentrated our analysis on the phase of the velocity oscillation, but the amplitude can also be measured and contains information on the speed of the possible coreforming motions. As illustrated by the cartoon in Fig. 12, the observed amplitude represents only the line-of-sight component of the true velocity amplitude, and it therefore needs to be corrected for the angle that the filament makes with the line of sight. Lacking a better estimate, we assume that each filament makes a 45 degree angle with the line of sight, and we use this value to derive representative velocities of the possible coreforming motions. As can be seen from Fig. 12, both filaments have velocity oscillations with an amplitude of approximately $0.04 \mathrm{~km} \mathrm{~s}^{-1}$, which would correspond to a projection-corrected value of approximately $0.06 \mathrm{~km} \mathrm{~s}^{-1}$. Interestingly, such subsonic speeds are similar to those inferred by Lee et al. (1999) for the inward motions towards a large number of low-mass dense cores, which were obtained using a very different technique (analysis of self-absorbed profiles). Also in agreement with previous core lifetime estimates (Lee \& Myers 1999), the time scale of collapse inferred from the $\gamma$ value in Eq. (6) is on the order of 0.5 Myr.

Even if the velocity oscillations in L1517 do not arise from contraction motions, their amplitude constrains core-formation models, as any underlying contraction motion has to be slower than about $0.05 \mathrm{~km} \mathrm{~s}^{-1}$ to remain undetected or confused with the observed oscillation. Thus, no matter how we interpret the observed velocity pattern, the conclusion that core formation in the L1517 cloud is strongly subsonic seems inescapable.

\section{How did the L1517 cores form?}

The picture that emerges from our analysis of L1517 is that core formation in this cloud has involved only subsonic motions and that it has started from conditions that were very close to hydrostatic equilibrium in an elongated configuration. These characteristics, together with the close fit of the filament density profiles by the model of an isothermal pressure-supported cylinder, suggest that some form of cylindrical gravitational fragmentation has played a role in the formation of the L1517 cores.

As discussed in Sect. 7.2, the simplest case of cylindrical fragmentation is that of an isothermal, infinitely-long, pressuresupported cylinder. Perturbation analysis shows that the gravitational fragmentation of such a system has a critical wavelength $\lambda_{\mathrm{f}}=3.94 H$, where $H$ is the filament width given by Eq. (2) (Stodólkiewicz 1963). Core formation via this simplest gravitational fragmentation model therefore requires that the dense cores are physically spaced by a distance grater than $\lambda_{\mathrm{f}}$.

To test whether the separation between cores in L1517 is consistent with the simplest gravitational fragmentation scenario, we have estimated the critical wavelength of the filaments using the $H$ values obtained from modeling their radial profiles (Table 2). These values imply that $\lambda_{\mathrm{f}}$ is about $1.2 \times 10^{18} \mathrm{~cm}$, or $570^{\prime \prime}$ for our assumed distance to Taurus (144 pc). As can be seen from Fig. 12, the observed distance between the cores in filaments 1 and 2 is $\approx 300^{\prime \prime}$, which is significantly less than the minimum value expected from the theory.

Assuming that the filaments are inclined with respect to the plane of the sky can change the above estimate, but it does not bring significant improvement. On the one hand, correcting for projection will increase the true distance between the cores, but it will also increase $\lambda_{\mathrm{f}}$ because the projection correction affects the column density estimate from which $\lambda_{\mathrm{f}}$ is derived (e.g., Arzoumanian et al. 2011). As a result, the observed separation between cores in the L1517 filaments seems to be about 1.52 smaller than predicted by the simplest model of gravitational fragmentation.

A number of factors can potentially explain the smaller-thanpredicted separation between cores in L1517. First of all, our $\lambda_{\mathrm{f}}$ value could have been overestimated. This is possible because $\lambda_{\mathrm{f}}$ was derived from the density analysis of the filaments, which depends on assuming a standard $\mathrm{C}^{18} \mathrm{O}$ abundance. If this molecule has suffered from large-scale freeze out in the filaments, the true gas volume density would be higher, and $\lambda_{\mathrm{f}}$ correspondingly smaller. A second possibility is that edge effects, due to the finite size of the filaments, are important in the outcome of the fragmentation, as suggested by the simulations of Bastien (1983) and Nelson \& Papaloizou (1993). Unfortunately, these effects depend very strongly on how sharp the filament edges are (Nelson \& Papaloizou 1993), and our data are not detailed enough to constrain this parameter. Finally, magnetic fields are known to affect the fragmentation of a cylinder (Nakamura et al. 1993, 1995; Gehman et al. 1996; Hanawa et al. 1993; Fiege \& Pudritz 2000b). Fiege \& Pudritz (2000b), in particular, show that both toroidal and poloidal magnetic fields decrease the critical length of fragmentation, and can do so by a significant factor for sufficiently strong fields. (They also decrease the instability growth rate.) Whether this could explain the observations of L1517 is unclear, as little is known about the magnetic field in the cloud. Kirk et al. (2006) find that the field towards core B is too weak to provide support, but these measurements are limited to a small region of the cloud already known to have undergone core formation. A detailed characterization of the large-scale magnetic field in the L1517 cloud is clearly needed.

Even if the L1517 cloud is less symmetric than assumed by our model, and its fragmentation history more complex than predicted by the simple theory, the observations presented here clearly constrain the process of core formation to have involved an elongated geometry and mostly subsonic motions. Core formation in this environment therefore appears to be a two-step process in which the elongated configuration close to 
equilibrium is formed first and subsonic fragmentation into cores occurs later. This two-step scenario of core formation has already been proposed by several authors based on different considerations, and it therefore seems to apply to more regions than just the one studied here (Schneider \& Elmegreen 1979; Hartmann 2002; Myers 2009). What our L1517 data show now is that the filamentary gas prior to fragmentation has a subsonic level of turbulence and a coherent velocity pattern, two properties that were thought before to only apply to dense cores. For such a quiescent state of the gas to occur, turbulent motions must have dissipated prior to (or rapidly during) filament formation, and therefore must play a limited role in the formation of the individual cores. If such a scenario is representative of core formation in other dark clouds, subsonic, velocity-coherent filaments must be a common feature, and embedded cores must systematically present kinematic patterns continuously connected to those of the large-scale filaments. Detailed study of the connection between dense cores and their surrounding cloud in a larger sample of regions should be able to test these two predictions.

\section{Summary}

We observed the $\mathrm{L} 1517$ dark cloud in $\mathrm{C}^{18} \mathrm{O}(1-0), \mathrm{N}_{2} \mathrm{H}^{+}(1-0)$, $\mathrm{SO}\left(J_{\mathrm{N}}=3_{2}-2_{1}\right)$ with the FCRAO telescope, and in the $1.2 \mathrm{~mm}$ dust continuum with the IRAM $30 \mathrm{~m}$ telescope. From the analysis of these data, we came to the following main conclusions.

1. The gas in the L1517 cloud is structured in four filaments with typical sizes of about $0.5 \mathrm{pc}$.

2. The radial profile of $\mathrm{C}^{18} \mathrm{O}(1-0)$ emission in each filament consists of a central flattened region and a power-law tail. An isothermal cylinder model provides an approximate fit to the emission, although better agreement is obtained with a softened power law profile.

3. Five starless cores are embedded in the filaments. Their chemical composition indicates that they are at different evolutionary stages, although their kinematic properties are very similar.

4. The velocity field of the gas in the L1517 cloud has been characterized by fitting Gaussians to the observed spectra. Most positions require one Gaussian, although a few regions require two, very likely due to the overlap between different components.

5. The filaments are extremely quiescent. Their nonthermal linewidth is subsonic and changes very little over the length of the filaments. The velocity centroids also change subsonically over the filament length. These characteristics indicate that the gas in the filaments is velocity coherent on scales of about $0.5 \mathrm{pc}$.

6. Although quiescent, the filaments have large-scale patterns of (subsonic) velocity. The gas in the dense cores follows the velocity pattern of the less dense gas closely, indicating that core formation has not decoupled the dense gas kinematically from its surrounding filament material.

7. In two filaments, the large-scale velocity patterns seem to consist of oscillations. A simple kinematic model shows that, at least in one filament, the oscillatory pattern is consistent with core-forming motions along the axis of the filament.

8. Core formation in L1517 seems to have occurred in two steps. First, the subsonic, velocity-coherent filaments have condensed out of the more turbulent ambient cloud. Then, the cores have fragmented quasi-statically and inherited the kinematics of their parental filament. Turbulence dissipation has therefore occurred on scales of $0.5 \mathrm{pc}$ or larger, and seems to have played little role in the formation of the individual cores.

Acknowledgements. We thank Mark Heyer for assistance with the FCRAO observations, Gilles Duvert for providing us with his old CO observations of the L1517 region, and Jouni Kainulainen for communicating his unpublished extinction map of L1517. We also thank an anonymous referee, Malcolm Walmsley, and Jens Kauffmann for a number of comments and suggestions that helped clarify the presentation. This research made use of NASA's Astrophysics Data System Bibliographic Services and the SIMBAD database, operated at the CDS, Strasbourg, France. It also made use of EURO-VO software, tools, and services. The EURO-VO is funded by the European Commission through contracts RI031675 (DCA) and 011892 (VO-TECH) under the 6th Framework Program and contracts 212104 (AIDA) and 261541 (VO-ICE) under the 7th Framework Program. The Digitized Sky Survey was produced at the Space Telescope Science Institute under US Government grant NAG W-2166. The images of these surveys are based on photographic data obtained using the Oschin Schmidt Telescope on Palomar Mountain and the UK Schmidt Telescope. The plates were processed into the present compressed digital form with the permission of these institutions.

\section{References}

Aikawa, Y., Herbst, E., Roberts, H., \& Caselli, P. 2005, ApJ, 620, 330 Alves, J., Lada, C. J., Lada, E. A., Kenyon, S. J., \& Phelps, R. 1998, ApJ, 506, 292

Alves, J. F., Lada, C. J., \& Lada, E. A. 2001, Nature, 409, 159

André, P., Men'shchikov, A., Bontemps, Si., et al. 2010, A\&A, 518, L102

Arzoumanian, D., André, Ph., Didelon, P., et al. 2011, A\&A, 529, L6

Ballesteros-Paredes, J., Klessen, R. S., \& Vázquez-Semadeni, E. 2003, ApJ, 592, 188

Barranco, J. A., \& Goodman, A. A. 1998, ApJ, 504, 207

Bastien, P. 1983, A\&A, 119, 109

Bastien, P., Arcoragi, J.-P., Benz, W., Bonnell, I., \& Martel, H. 1991, ApJ, 378, 255

Beichman, C. A., Myers, P. C., Emerson, J. P., et al. 1986, ApJ, 307, 337

Benson, P. J., \& Myers, P. C. 1989, ApJS, 71, 89

Bergin, E. A., \& Langer, W. D. 1997, ApJ, 486, 316

Bergin, E. A., \& Tafalla, M. 2007, ARA\&A, 45, 339

Bergin, E. A., Alves, J., Huard, T., \& Lada, C. J. 2002, ApJ, 570, L101

Bernes, C. 1979, A\&A, 73, 67

Binney, J., \& Tremaine, S. 1987, Galactic Dynamics (Princeton University Press) Burkert, A., \& Hartmann, L. 2004, ApJ, 616, 288

Caselli, P., Walmsley, C. M., Tafalla, M., Dore, L., \& Myers, P. C. 1999, ApJ, 523, L165

Caselli, P., Benson, P. J., Myers, P. C., \& Tafalla, M. 2002, ApJ, 572, 238

Cazzoli, G., Dore, L., Puzzarini, C., \& Beninati, S. 2002, Phys. Chem. Chem. Phys., 4, 3575

Cazzoli, G., Puzzarini, C., \& Lapinov, A. V. 2003, ApJ, 592, L95

Chandrasekhar, S. 1951, Roy. Soc. London Proc. Ser. A, 210, 26

Crapsi, A., Caselli, P., Walmsley, C. M., et al. 2005, ApJ, 619, 379

Crutcher, R. M., Wandelt, B., Heiles, C., Falgarone, E., \& Troland, T. H. 2010, ApJ, 725, 466

di Francesco, J., Evans, N. J., II, Caselli, P., et al. 2007, Protostars and Planets V, ed. B. Reipurth, D. Jewitt, \& K. Keil (Tucson: Uni. Arizona Press), 17

Duvert, G., Cernicharo, J., \& Baudry, A. 1986, A\&A, 164, 349

Enoch, M. L., Young, K. E., Glenn, J., et al. 2006, ApJ, 638, 293

Fiege, J. D., \& Pudritz, R. E. 2000a, MNRAS, 311, 85

Fiege, J. D., \& Pudritz, R. E. 2000b, MNRAS, 311, 105

Frerking, M. A., Langer, W. D., \& Wilson, R. W. 1982, ApJ, 262, 590

Gehman, C. S., Adams, F. C., \& Watkins, R. 1996, ApJ, 472, 673

Goodman, A. A., Benson, P. J., Fuller, G. A., \& Myers, P. C. 1993, ApJ, 406, 528

Goodman, A. A., Barranco, J. A., Wilner, D. J., \& Heyer, M. H. 1998, ApJ, 504 223

Hanawa, T., Nakamura, F., Matsumoto, T., et al. 1993, ApJ, 404, L83

Hartmann, L. 2002, ApJ, 578, 914

Heyer, M. H., Vrba, F. J., Snell, R. L., et al. 1987, ApJ, 321, 855

Hirota, T., Ohishi, M., \& Yamamoto, S. 2009, ApJ, 699, 585

Inutsuka, S.-I., \& Miyama, S. M. 1992, ApJ, 388, 392

Inutsuka, S.-I., \& Miyama, S. M. 1997, ApJ, 480, 681

Johnstone, D., Fiege, J. D., Redman, R. O., Feldman, P. A., \& Carey, S. J. 2003, ApJ, 588, L37

Kenyon, S. J., Gómez, M., \& Whitney, B. A. 2008, Handbook of Star Forming Regions, Vol. I, ed. B. Reipurth, 405

Kirk, J. M., Ward-Thompson, D., \& André, P. 2005, MNRAS, 360, 1506 
Kirk, J. M., Ward-Thompson, D., \& Crutcher, R. M. 2006, MNRAS, 369, 1445 Kirk, H., Johnstone, D., \& Tafalla, M. 2007a, ApJ, 668, 1042

Kirk, J. M., Ward-Thompson, D., \& André, P. 2007b, MNRAS, 375, 843

Klessen, R. S., Ballesteros-Paredes, J., Vázquez-Semadeni, E., \& Durán-Rojas, C. 2005, ApJ, 620, 786

Kuiper, T. B. H., Langer, W. D., \& Velusamy, T. 1996, ApJ, 468, 761

Ladd, E. F., \& Myers, P. C. 1991, Atoms, Ions and Molecules: New Results in Spectral Line Astrophysics (San Francisco: ASP), 16, 241

Larson, R. B. 1981, MNRAS, 194, 809

Larson, R. B. 1985, MNRAS, 214, 379

Lemme, C., Wilson, T. L., Tieftrunk, A. R., \& Henkel, C. 1996, A\&A, 312, 585

Lee, C. W., \& Myers, P. C. 1999, ApJS, 123, 233

Lee, C. W., Myers, P. C., \& Tafalla, M. 1999, ApJ, 526, 788

Luhman, K. L., Mamajek, E. E., Allen, P. R., \& Cruz, K. L. 2009, ApJ, 703, 399

Lynds, B. T. 1962, ApJS, 7, 1

Mouschovias, T. C., \& Ciolek, G. E. 1999, in The Origin of Stars and Planetary Systems, ed. C. J. Lada, \& N. D. Kylafis (Kluwer), 305

Myers, P. C. 1983, ApJ, 270, 105

Myers, P. C. 2009, ApJ, 700, 1609

Myers, P. C., \& Benson, P. J. 1983, ApJ, 266, 309

Myers, P. C., Fuller, G. A., Goodman, A. A., \& Benson, P. J. 1991, ApJ, 376, 561

Nachman, P. 1979, ApJS, 39, 103

Nagasawa, M. 1987, Progress of Theoretical Physics, 77, 635

Nakamura, F., Hanawa, T., \& Nakano, T. 1993, PASJ, 45, 551
Nakamura, F., Hanawa, T., \& Nakano, T. 1995, ApJ, 444, 770 Nelson, R. P., \& Papaloizou, J. C. B. 1993, MNRAS, 265, 905 Ostriker, J. 1964, ApJ, 140, 1056

Padoan, P., Juvela, M., Goodman, A. A., \& Nordlund, Å. 2001, ApJ, 553, 227

Pagani, L., Daniel, F., \& Dubernet, M.-L. 2009, A\&A, 494, 719

Pineda, J. E., Goodman, A. A., Arce, H. G., et al. 2010, ApJ, 712, L116

Schneider, S., \& Elmegreen, B. G. 1979, ApJS, 41, 87

Shu, F. H., Adams, F. C., \& Lizano, S. 1987, ARA\&A, 25, 23

Stepnik, B., Abergel, A., Bernard, J.-P., et al. 2003, A\&A, 398, 551

Stodólkiewicz, J. S. 1963, Acta Astron., 13, 30

Strom, K. M., Strom, S. E., \& Vrba, F. J. 1976, AJ, 81, 320

Tafalla, M., Myers, P. C., Caselli, P., Walmsley, C. M., \& Comito, C. 2002, ApJ, 569,815

Tafalla, M., Myers, P. C., Caselli, P., \& Walmsley, C. M. 2004, A\&A, 416, 191

Tafalla, M., Santiago-García, J., Myers, P. C., et al. 2006, A\&A, 455, 577

van den Ancker, M. E., de Winter, D., \& Tjin A Djie, H. R. E. 1998, A\&A, 330, 145

Vázquez-Semadeni, E., Kim, J., Shadmehri, M., \& Ballesteros-Paredes, J. 2005, ApJ, 618, 344

Walsh, A. J., Myers, P. C., \& Burton, M. G. 2004, ApJ, 614, 194

Ward-Thompson, D., André, P., Crutcher, R., et al. 2007, Protostars and Planets V, ed. B. Reipurth, D. Jewitt, \& K. Keil (Tucson: Uni. Arizona Press), 33

Zhou, S., Wu, Y., Evans, N. J., II, Fuller, G. A., \& Myers, P. C. 1989, ApJ, 346, 168 